\title{
An experimental study on the efficiency of transpiration cooling in laminar and turbulent hypersonic flows
}

\author{
A. Gülhan · S. Braun
}

Received: 8 February 2010/Revised: 30 June 2010/Accepted: 16 July 2010/Published online: 14 August 2010

(C) The Author(s) 2010. This article is published with open access at Springerlink.com

\begin{abstract}
An experimental study on the efficiency of transpiration cooling in hypersonic laminar and turbulent flow regimes is carried out in the Hypersonic Windtunnel Cologne with a focus on the aerothermal problems downstream of the cooled model part. The model is made of a material of low thermal conductivity (PEEK) with an integrated probe of a porous material. The experimental setup allows the direct comparison of the thermal behavior of transpiration cooling to a well-defined and radiatively cooled reference surface. Experiments are performed at Mach number of 6 and two different Reynolds numbers. Air, argon and helium are used as coolants at various flow rates, in order to identify the influence of coolant medium on cooling efficiency. The cooling efficiency of air and argon is comparable. Helium provides significantly higher cooling efficiency at the same blowing ratio, i.e. same coolant mass flow rate. The experimental data shows that the efficiency of the transpiration cooling in turbulent flows is much lower than in laminar flow.
\end{abstract}

\section{List of symbols \\ A Area $\left[\mathrm{m}^{2}\right]$ \\ $c_{p} \quad$ Pressure coefficient [-] \\ $H 2 K \quad$ Hypersonic Wind Tunnel Cologne \\ $M \quad$ Mach number [-] \\ $p \quad$ Pressure $\left[\mathrm{N} \mathrm{m}^{-2}\right]$ \\ PEEK Poly ether ether ketone \\ PSI Pressure Systems Incorporated}

A. Gülhan $(\bowtie)$

German Aerospace Center (DLR), Cologne, Germany

e-mail: ali.guelhan@dlr.de

URL: www.dlr.de

S. Braun

RWTH Aachen University, Aachen, Germany

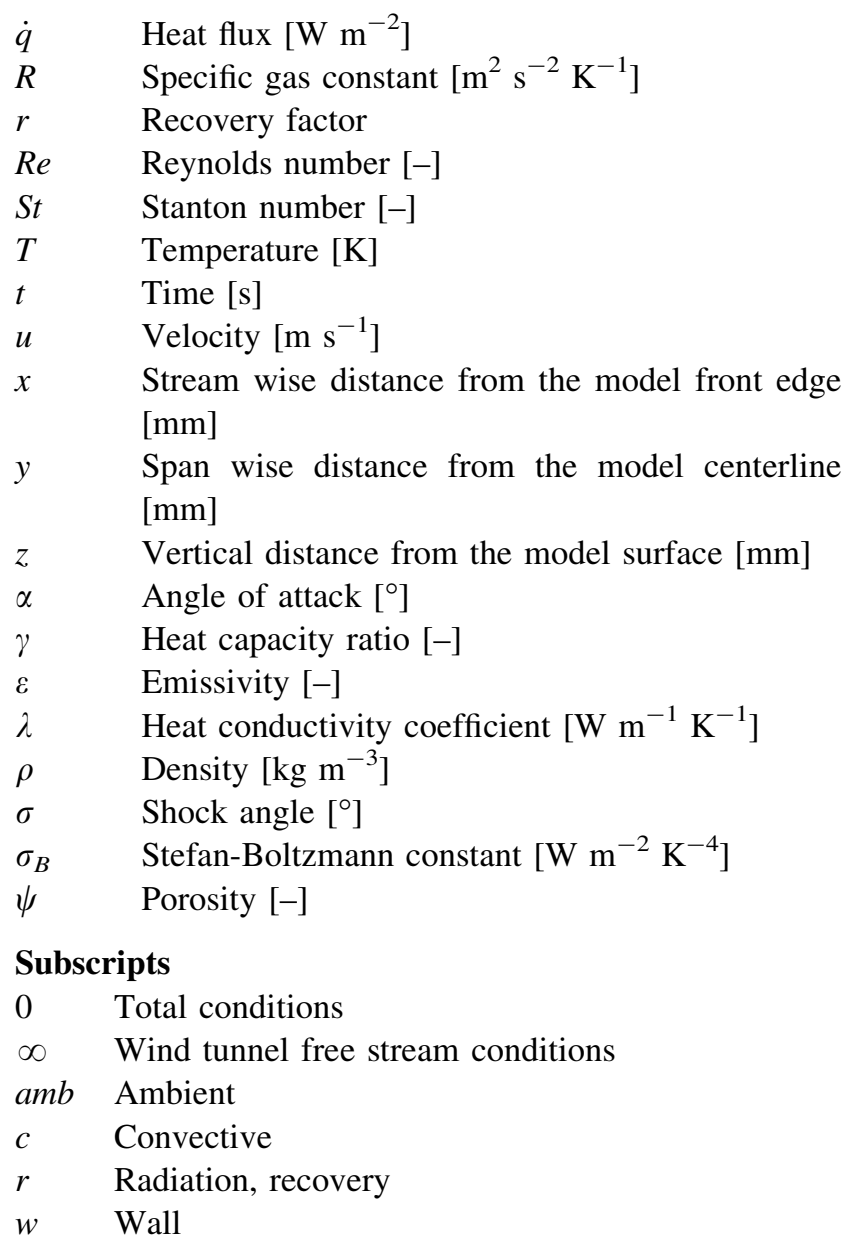

\section{Introduction}

Some components of atmospheric entry vehicles or hypersonic space planes are partially exposed to severe 
aerothermal loads. Depending on the trajectory, these components may need a sophisticated thermal protection which is commonly based on ceramic matrix composite materials. Such materials have capability to sustain very high temperature. This property is used to protect the vehicle's interior from being heated, since at high surface temperature most of the huge convective heat that is generated by the hypersonic flow is radiated back to space leaving only a small fraction penetrating the surface. This passive protection philosophy, however, is limited by the surface materials' operational limit temperature. In general, these limits are partially exceeded for planetary entries and Earth return. For some flight trajectories of a hypersonic space plane, high mechanical and dynamic loads are present in addition to the aerothermal loads. Most of the thermal protection materials or hot structures including ablative Thermal Protection System (TPS) cannot sustain such combined severe loads. Therefore, such missions can only be realized by extending the capabilities of hot structures using active cooling.

Transpiration cooling is a very promising active cooling concept. The coolant is a gas that effuses through a porous surface material to the outside without disturbing the external flow field considerably. In 1956, Rubesin performed a study for the gas injection into the high-speed turbulent boundary layer and discussed its influence on the heat transfer and skin friction coefficients (Rubesin 1956). He mentioned the problem of the tripping of the laminar boundary layer by a gas injection and therefore concentrated his work into the turbulent flow. Although the Rubesin's analytical results of cooling efficiency showed a reasonable agreement with the experimental data of a flat plate in a limited Mach number range, he could not provide a conclusive result on the effect of different gases as coolant. Pappas et al. carried out experiment an experimental study on a cone with transpiration cooling to clarify effects of Mach number on the efficiency of transpiration cooling with different gases as coolant (Rubesin and Pappas 1964). In contrast to the theory of Rubesin and Pappas (1958), they could not measure any remarkable Stanton number decrease with increasing Reynolds number at
Mach number of 0.7. At supersonic Mach numbers and for small and moderate blowing ratios, they measured the same small influence of the Mach number on the heat flux coefficient as predicted by Rubesin. This work showed also that the theory of Rubesin cannot be applied for high blowing ratios, at which a homogenous gas injection through the porous material is violated. Further similar fundamental studies on transpiration cooling showed the big potential of this technique for its application on turbine blades and combustion chambers (Luikov 1963; Kays 1972). The capabilities could significantly be extended with porous ceramic composite materials (CMC). At DLR, a transpiration cooling concept based on porous carbon reinforced carbon $(\mathrm{C} / \mathrm{C})$ was developed and successfully applied to combustion chamber cooling in rocket engines (Lezuo 1998; Serbest 2002; Ghadiani 2005).

Recent studies at DLR aimed to apply this concept in combination with new generation CMC materials for atmospheric entry flights (Kuhn and Hald 2008). It has to be mentioned that in contrast to the mainly turbulent flow in gas turbines and combustion chambers, the boundary layer flow during atmospheric entry pass all laminar, transitional and turbulent regimes. Therefore, a detailed experimental study has been carried out on the applicability of transpiration cooling first at high-altitude atmospheric entry conditions, i.e. low laminar Reynolds numbers (Esser et al. 2007). In the frame of this study experiments in the DLR arc-heated facilities, L2K and L3K on porous ceramic matrix composite materials using gas as coolant showed significant decrease in the surface temperature, i.e. heat flux rate ${ }^{10}$. As shown in Fig. 1, the surface temperature of transpiration cooled porous sample consisting of carbon fiber-reinforced carbon material decreases significantly compared to the un-cooled reference surface, which is made of the same material with negligible porosity, i.e. without cooling. Depending on the coolant mass flow ratio, a temperature decrease about $50-65 \%$ was measured. Further experiments in the L2K facility using water and gas as coolant also demonstrated the feasibility of the transpiration cooling using water (Van Foreest et al. 2007). However, all these experiments were carried out in laminar
Fig. 1 IR image (left) and surface temperature history of spots (right) during transpiration cooling tests in arc-heated facility L3K (Esser et al. 2007)
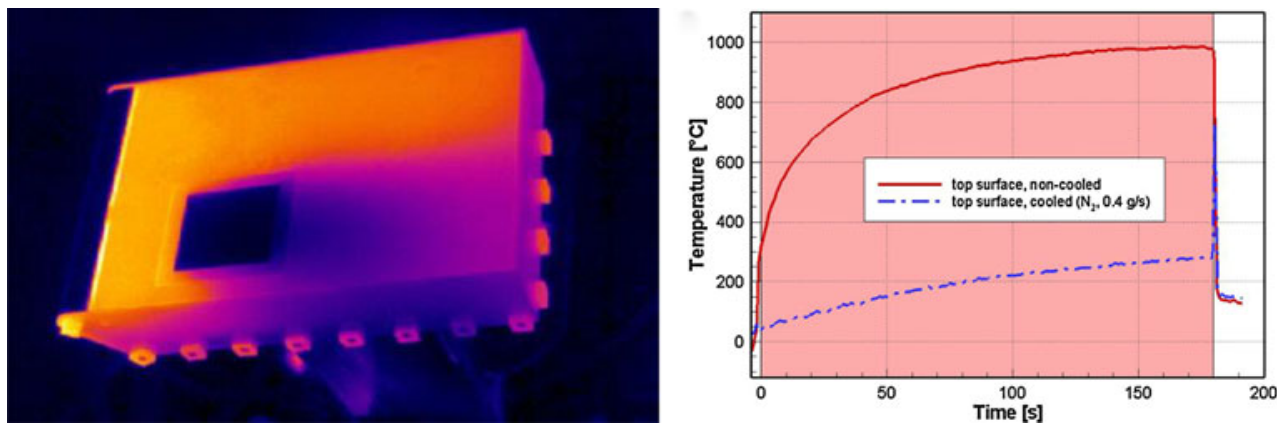
flows at very low laminar Reynolds number of less than 100,000 , at which boundary layer stays always laminar downstream of the porous sample, where a film cooling establishes. The main advantage of recent studies in comparison works in the past is the availability of IR thermography, which allows to measure two dimensional surface temperature distribution.

In the previous study, we studied the influence of the boundary layer condition on the efficiency of a cooling with distinct holes or coolant injection slots for hypersonic flows in the DLR hypersonic windtunnel H2K (Esser and Gülhan 2008). Experiments were carried out in the DLR hypersonic windtunnel $\mathrm{H} 2 \mathrm{~K}$ at Reynolds numbers, which were two orders of magnitude higher compared to the arc-heated facility L3K. This study showed clearly that at sufficiently high laminar Reynolds numbers, the flow downstream of the cooling hole or slot can be tripped leading to local overheating of the model surface (Fig. 2). Similar experiments were performed by Heufer and Olivier (2008) in a shock tunnel with a high enthalpy flow field. Despite some additional information with respect to the influence of the ratio of surface temperature to total temperature on the cooling efficiency, their experiments provided similar behavior concerning the Reynolds number effects.

Dedicated comparative experimental works on the efficiency of the transpiration cooling in both turbulent and laminar flow regimes at typical atmospheric entry flight conditions are quite rare. Therefore, the main objective of this work was to contribute for closing this gap.

This paper first describes the main experimental tools, namely, the DLR hypersonic windtunnel $\mathrm{H} 2 \mathrm{~K}$, test model and instrumentation. The flow parameters and measured surface temperature distribution will follow this part. In addition, the algorithm used for the heat flux determination from the measured surface temperature will be described. The experimental results and main findings will be the main elements of the paper.

\section{Experimental tools and test parameters}

\subsection{Hypersonic windtunnel $\mathrm{H} 2 \mathrm{~K}$}

The experiments are performed at the DLR Hypersonic Wind Tunnel (H2K) in Cologne. Figure 3 shows the H2K, which is an intermittent blow-down tunnel for hypersonic flows. It uses contoured, axially symmetrical, and replaceable nozzles. For aerodynamic testing, nozzles for Mach numbers of 4.8, 5.3, 6.0, 7.0, 8.7, and 11.2 are available. The diameter of the nozzles is $0.6 \mathrm{~m}(0.36 \mathrm{~m}$ at $M_{\infty}=4.8$ ). In order to achieve the pressure ratio required to build up a jet flow for approximately $30 \mathrm{~s}$, the pressure in the test chamber is reduced by means of a vacuum sphere. Due to the intense expansion of the air in the nozzle, its static temperature decreases significantly. To prevent condensation of air particles and for tests at high temperatures, the air is preheated by an electric heater. Eight electric heaters with a maximum electrical power of $5 \mathrm{MW}$ heat the air up to $1,100 \mathrm{~K}$. The maximum total pressure is 55 bar. The performance map of $\mathrm{H} 2 \mathrm{~K}$ is given in Fig. 3.

The windtunnel flow conditions for these tests are summarized in Table 1; the measurement accuracy of the main wind tunnel operation parameters is: $\Delta M / M=0.5 \%$, $\Delta T_{0} / T_{0}=0.75 \%$, and $\Delta p_{0} / p_{0}=0.02 \%$.

\subsection{Test model}

The model design is based on the model tested in the archeated facility L3K (Esser et al. 2007). The setup that is used for the test campaign in the hypersonic windtunnel $\mathrm{H} 2 \mathrm{~K}$ is shown in Fig. 4. The complete setup is installed on top of a metallic model holder. The model is $59 \mathrm{~mm}$ height and has an overall width of $192 \mathrm{~mm}$. It has a length of $297.9 \mathrm{~mm}$ (Fig. 2). A metallic porous sample is integrated into the model on top of the gas reservoir, where the
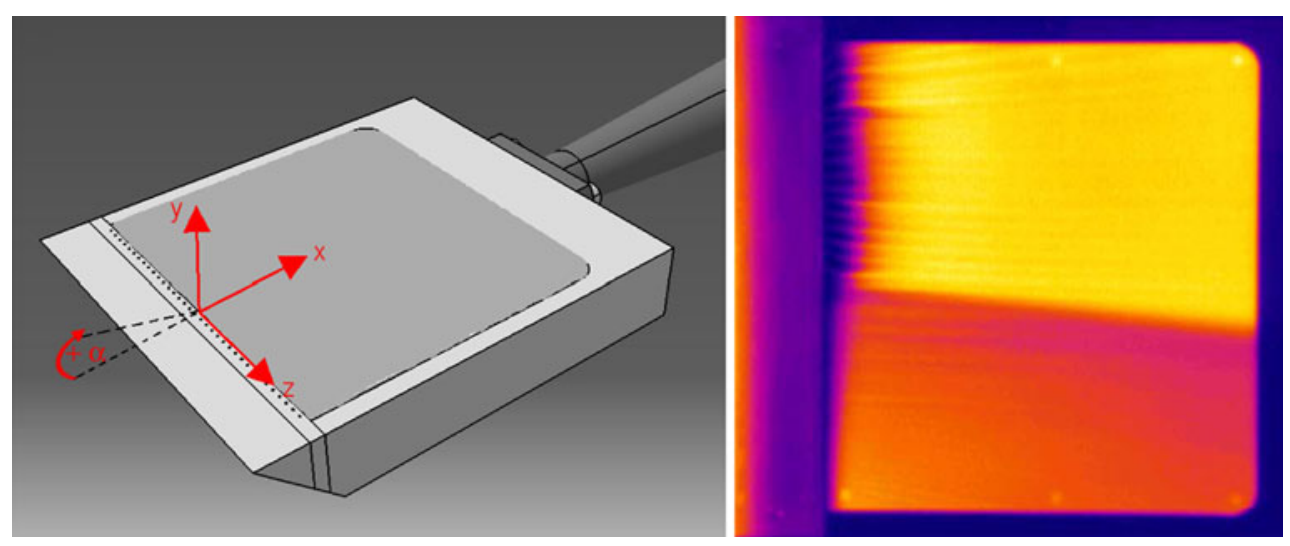

Fig. 2 Active cooling system with tangential air cooling injection in the hypersonic windtunnel H2K (left) and its IR image (right) (Esser and Gülhan 2008) 
Fig. 3 Experimental set-up (left) and the performance map of $\mathrm{H} 2 \mathrm{~K}$ (right)
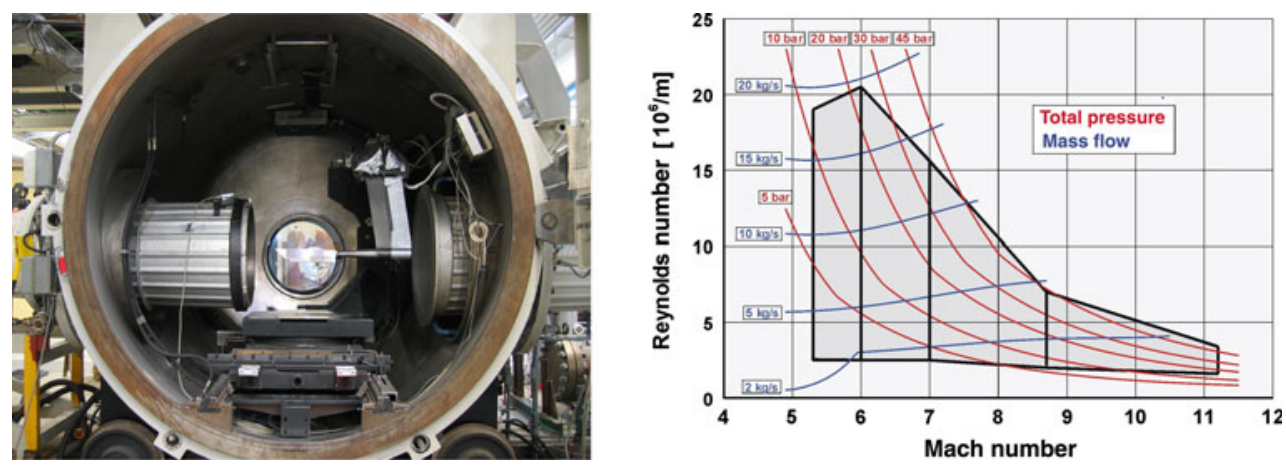

Table 1 Wind tunnel flow conditions

\begin{tabular}{lll}
\hline & $\begin{array}{l}\text { Flow } \\
\text { condition I }\end{array}$ & $\begin{array}{l}\text { Flow } \\
\text { condition II }\end{array}$ \\
\hline Free stream Mach number, $M_{\infty}[-]$ & 6 & 6 \\
Free stream pressure, $p_{\infty}[\mathrm{Pa}]$ & 380 & 1,045 \\
Free stream temperature, $T_{\infty}[\mathrm{K}]$ & 89 & 57 \\
Free stream density, $\rho_{\infty}\left[\mathrm{kg} / \mathrm{m}^{3}\right]$ & 0.01487 & 0.06352 \\
Dynamic pressure, $q[\mathrm{~Pa}]$ & 9,576 & 26,335 \\
Total temperature, $T_{0}[\mathrm{~K}]$ & 730 & 470 \\
Total pressure, $p_{0}[\mathrm{~Pa}]$ & $6 \times 10^{5}$ & $16.5 \times 10^{5}$ \\
Reynolds number, $R e[-]$ & $0.73 \times 10^{6}$ & $4.56 \times 10^{6}$ \\
\hline
\end{tabular}

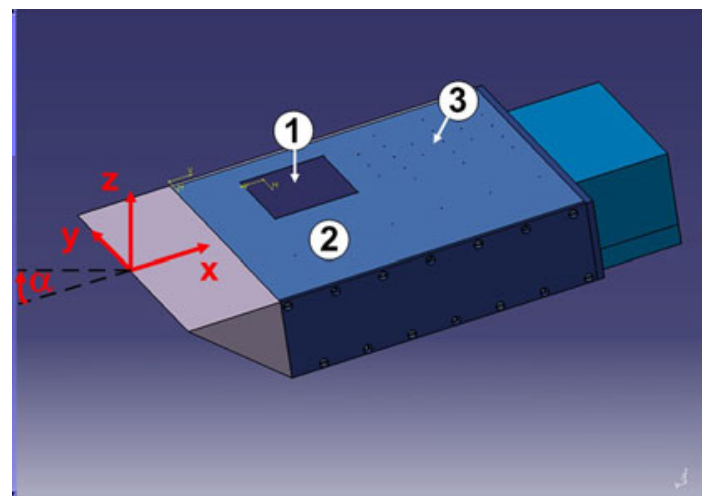

Fig. 4 A schematic view of the test model with the coordinate system: (1) porous sample, (2) PEEK plate, (3) pressure ports

pressure and temperature of the coolant gas are monitored. The 61-mm-wide square porous sample is made of Inconel 625. It consists of $\mathrm{Ni}(63.2 \%), \mathrm{Cr}(22.3 \%), \mathrm{Mo}(9.1 \%)$, and $\mathrm{Nb}(3.4 \%)$. It has a volumetric porosity of $61.99 \%$ and a thickness of $6.5 \mathrm{~mm}$. Its density is $2.79 \mathrm{~kg} / \mathrm{m}^{3}$.

In order to determine the heat flux distribution from measured surface temperature data with IR thermography, the main model upper part is made of polyether ether ketone (PEEK). It is a low conductive material and provides the correct conditions for the semi-infinite wall assumption (Henckels and Gruhn 2005; Häberle and Gülhan 2007).
Since the research focuses on the determination and interpretation of the heat transmission on the surface of the model, the material of the model must meet specific requirements. Thus, for a convective heat flux determination, the unsteady-state heat conduction equation is considered to be one dimensional, i.e., the aspect of cross-heat conduction is neglected. A material that fulfills this requirement is polyether ether ketone (PEEK), given its low thermal conductivity. PEEK is a linear aromatic polymer of high mechanical resistance. Apart from its ability to withstand chemical attack and hydrolysis, it qualifies for use in aerothermodynamic research particularly due to its thermal properties at high temperatures. Key characteristics to be emphasized in this context are its relatively high melting temperature of $616 \mathrm{~K}$, its maximum continuous temperature of $533 \mathrm{~K}$, and its low thermal conductivity of $\lambda=0.258 \mathrm{~W} \mathrm{~m}^{-1} \mathrm{~K}^{-1}$. Another boundary condition to be ensured is the assumed semi-infinite wall thickness of the model. In other words, the wall thickness must be chosen such that the heat flux applied to the model surface will not be noticeable on the underside during the time of the test.

\subsection{Measurement techniques}

The wall pressures on the PEEK model are measured with the standard PSI system. The pressure taps are connected to PSI modules (PSI 8400 system). The static accuracy of the PSI modules is $\pm 0.1 \%$ of the full-scale deflection. Measurement errors due to Mach number deviations and changes in the free-stream angle are generally greater. For the flow visualization in $\mathrm{H} 2 \mathrm{~K}$, a coincidence Schlieren system is used. The Schlieren images are focused directly into a camera.

The signal detected by a thermo camera depends mainly on the temperature $T$ of the thermographed body and its emission coefficient. The ThermaCAM SC 3000 camera system employed for our purpose uses a QWIP detector with $320 \times 240$ pixels and $60 \mathrm{~Hz}$, operating in the longwave infrared range $(8-9 \mu \mathrm{m})$ at temperatures between -20 and $2,000^{\circ} \mathrm{C}$. The camera has different calibrated 
measurement ranges. During this test campaign, the measurement range of $10-150^{\circ} \mathrm{C}$ is used. The variance of the emission coefficient is negligible in this temperature range. The emission coefficient of the PEEK model is $\varepsilon=0.95$. An estimate of the measurement error caused by the angle of the camera's optical axis to a reference line vertical to the model yields a minor deviation of $1 \%$ at angles of observation below $50^{\circ}$.

\section{Determination of the heat flux rate and cooling efficiency}

\subsection{Heat flux rate determination}

In literature, several algorithms are given to derive heat flux data from surface temperature measurements. Unfortunately, most of these techniques cannot be perfectly adapted to the application at blow down facilities, which show radiation effects and significantly rising wall temperatures. Further on, in order to reduce lateral heat transfer, thick wall models are generally built of artificial resin with low thermal conductivity and temperaturedependent material properties. The heat flux evaluation presented here requires a more advanced approach, starting from the thermal energy balance on a solid volume element and leads to the one-dimensional nonlinear heat equation (Henckels and Gruhn 2005).

$\frac{\partial T}{\partial t}=a_{(T)} \cdot \frac{\partial^{2} T}{\partial z^{2}}+b_{(T)} \cdot\left(\frac{\partial T}{\partial z}\right)^{2}$

with the thermal diffusivity

$a_{(T)}=\frac{\lambda_{(T)}}{\rho_{(T)} \cdot c_{p(T)}}$

and

$b_{(T)}=\frac{\frac{d \lambda_{(T)}}{d T}}{\rho_{(T)} \cdot c_{p(T)}}$

This differential temperature expression is a function not merely of place and time, but also of the density $\rho$, the thermal conductivity $\lambda$, and the specific heat capacity $c_{p}$. Therefore, an accurate knowledge of material characteristics and their behavior under temperature variation is essential. This study uses the temperature-dependent material parameters of PEEK, which are described in the former paper (Gülhan et al. 2008).

To calculate the temperature profile inside the model wall, Eq. 1 can be solved by an explicit finite-difference procedure by marching stepwise through the time $t=k \Delta t$. For numerical stability, the Courant-FriedrichsLewy condition is postulated as follows
$\Delta t \leq \frac{\Delta z^{2}}{2 \cdot a_{(T)}}$

If Eq. 4 holds, the unknown temperature $T_{i, k+1}$ (the index $i$ describes equally spaced points in $z$-direction normal to the surface) at the next time step is calculated simply by

$$
\begin{aligned}
T_{i, k+1}= & T_{i, k}+\frac{\Delta t}{\Delta z^{2}} a_{\left(T_{i, k}\right)}\left(T_{i-1, k}-2 T_{i, k}+T_{i+1, k}\right) \\
& +\frac{\Delta t}{\Delta z^{2}} \frac{b_{\left(T_{i, k}\right)}}{4}\left(T_{i-1, k}-T_{i+1, k}\right)^{2} .
\end{aligned}
$$

As start condition $(t=0)$, a homogeneous temperature distribution inside the model is assumed, i.e. for all points the initial temperature $T_{i, 0}$ is set equal to the surface temperature $T_{0,0}$. The surface temperatures $T_{0, k}$ are measured by the infrared system as the boundary condition at the surface. A second boundary condition inside the wall at

$z \geq 4 \cdot \sqrt{a_{\max } \cdot t_{\max }}$

is based on the assumption of an adiabatic situation, i.e. $T_{i+1, k+1}=T_{i, k+1}$. This numerical procedure provides temperature profiles $T_{i}$ inside the model wall at each time step $k$. Based on these profiles, the convective heat flux $q_{c}$ between the flow and the wall can now be derived from the heat flux balance at the surface:

$\dot{q}_{c}=\dot{q}_{r}+\dot{q}_{w}$

The radiative heat flux $q_{r}$ between the large surface of the test section, which is assumed as staying at ambient temperature $T_{a m b}$, and the model surface is given by the Stefan-Boltzmann law

$\dot{q}_{r}=\varepsilon \cdot \sigma\left(T_{(0, k)}^{4}-T_{a m b}^{4}\right)$.

The heat flux $q_{w}$ absorbed by the wall is given by Fourier's law

$\dot{q}_{w}=\left.\lambda_{\left(T_{0, k}\right)} \cdot \frac{d T}{d z}\right|_{z=0}$

which is solved by a high order interpolation scheme for the differentiation at the surface. PEEK is a suitable model material for tests in which surface temperatures do not exceed $500 \mathrm{~K}$.

This thermal data evaluation technique proves to be an efficient tool to resolve in stationary heat flux loads with an error of less than $8 \%$, as being typical on models used in blow down facilities. In contrast to existing data evaluation procedures, it is not mandatory to realize a sudden heat load at a constant level or a constant heat transfer coefficient, respectively. Special technical effort to control the starting process of the tunnel flow is not required any more.The resulting Stanton number characterizing the convective heat transmission has been related to the free stream flow conditions by 
$S t=\frac{\dot{q}_{c}}{\rho_{\infty} u_{\infty} c_{p}\left(T_{r}-T_{i=0, k}\right)}$

where $\dot{q}_{c}$ is convective heat flux rate, $\rho_{\infty}$ is the upstream density, $u_{\infty}$ is the upstream velocity and $c_{p}$ is the heat capacity. $T_{i=0}$ is the wall temperature of the model. $T_{i=0, k}$ is determined by the infrared measurements. The recovery temperature is defined as $T_{r}=r T_{0}$ with the reservoir temperature $T_{0}$ and the recovery factor $r=0.91$, as proposed by Delery (1988).

For the convective heat flux calculation, the uncertainty of the measured absolute temperature value is non critical, while the temperature gradient over time is the most important parameter. A sensitivity analyses has been performed for the Stanton number accuracy using PEEK material data and wind tunnel conditions. Like former similar studies with respect to the IR application on PEEK models, the overall accuracy of the Stanton number is about $\pm 20 \%$ (Häberle and Gülhan 2007).

\subsection{Main parameters of transpiration cooling}

The transpiration cooling can be described with the two characteristic numbers, which are the cooling efficiency $\eta$ and the blowing ratio $F$. Because of the limited testing time of $30 \mathrm{~s}$ in $\mathrm{H} 2 \mathrm{~K}$, the adiabatic wall temperature cannot be reached. In addition, the total temperature of the coolant remains almost constant. Therefore, in this work the cooling efficiency is defined using Stanton number ratio for the flow with and without cooling:

$\eta=1-\frac{S t_{c}}{S t_{n c}}$

The blowing ratio $F$ is defined as the ratio of the specific coolant mass flow rate and the boundary layer mass flow rate:

$F=\frac{\dot{m}_{c} / A_{c}}{\dot{m}_{e} / A_{e}}=\frac{\rho_{c} u_{c}}{\rho_{e} u_{e}}$

where $e$ denotes the boundary layer edge conditions, which are determined from numerical computation for both laminar and turbulent flow cases. The cross-sectional area of the porous surface for the coolant $A_{c}$ can be defined as a function of the porosity $\psi$;
$A_{c}=\psi \cdot A_{\text {probe }}$

For the calculation of the blowing ratio $\mathrm{F}$ besides measured coolant flow rate, the mass flow rate of the cross flow in the boundary layer has to be determined. Therefore, for both FC I and FC II test conditions the flow field was calculated using the commercial CFD code Fluent (2003). Table 2 shows the computed parameter at the upstream edge of the porous sample $(x=108 \mathrm{~mm})$. For these calculations, a coolant mass flow rate of $1 \mathrm{~g} / \mathrm{s}$ is used.

\section{Results}

\subsection{Heat flux measurements at angle of attack of $-5^{\circ}$}

First tests have been carried out at low Reynolds number condition (FC 1), at which the flow is laminar. The coolant mass flow rate is set to $0.2 \mathrm{~g} / \mathrm{s}$ and $0.4 \mathrm{~g} / \mathrm{s}$, which correspond to blowing ratios of $8.48 \times 10^{-3}$ and $16.95 \times 10^{-3}$, respectively. As coolant air, argon and helium are used. As mentioned before, the technique at $\mathrm{H} 2 \mathrm{~K}$ allows determining the time history of the surface temperature and heat flux rate, i.e. Stanton number. Figure 5 shows the data of two spots, which are called SP1 and SP2. SP1 is located on the un-cooled reference side of the model, while SP2 is a spot just downstream of the cooled porous sample. For the surface temperature measurement with IR thermograpy, the emissivity value of PEEK (0.95) is used. Before starting the windtunnel, the model is on the flow axis and the cooling is on. Therefore, there is a slight difference between the temperatures of two spots in the beginning of the test. The flow establishment takes about 3-4 s. During this phase, the model is exposed to a not well-defined flow field. But after this time, the flow has a stationary character. The Stanton number gets constant after about $8 \mathrm{~s}$ testing time. In order to eliminate any effects coming from transitional time of the facility operation, all experiments are evaluated for the test point of $10 \mathrm{~s}$. The transpiration cooling through the porous metallic sample leads to significantly lower surface temperature and Stanton number on the PEEK material.

Figure 6 measured surface temperature distributions and heat flux profiles along two lines on each cooled part and

Table 2 Calculated blowing ratio values

\begin{tabular}{lllllrr}
\hline Flow condition & $\alpha\left[{ }^{\circ}\right]$ & $\operatorname{Re}\left[/ 10^{6}\right]$ & Boundary layer & $\delta[\mathrm{mm}]$ & $\rho \mathrm{u}\left[\mathrm{kg} /\left(\mathrm{m}^{2} \mathrm{~s}\right)\right]$ & $\begin{array}{l}F(m=1 \mathrm{~g} / \mathrm{s}) \\
{[-]\left(\times 10^{-3}\right)}\end{array}$ \\
\hline FC I & -5 & 0.73 & Laminar & 2.061 & 10.23 & 42.38 \\
FC II & -5 & 4.56 & Laminar & 1.161 & 28.73 & 15.08 \\
FC I & -20 & 0.73 & Laminar & 1.245 & 29.69 & 14.60 \\
FC II & -20 & 4.56 & Turbulent & 2.054 & 126.11 & 3.44 \\
\hline
\end{tabular}


Fig. 5 Measured surface temperature distribution (left) and time history of temperature and Stanton number of two spots (right) during test at angle of attack of $-5^{\circ}$ and FC 1 with air as coolant for $F=16.95 \times 10^{-3}$
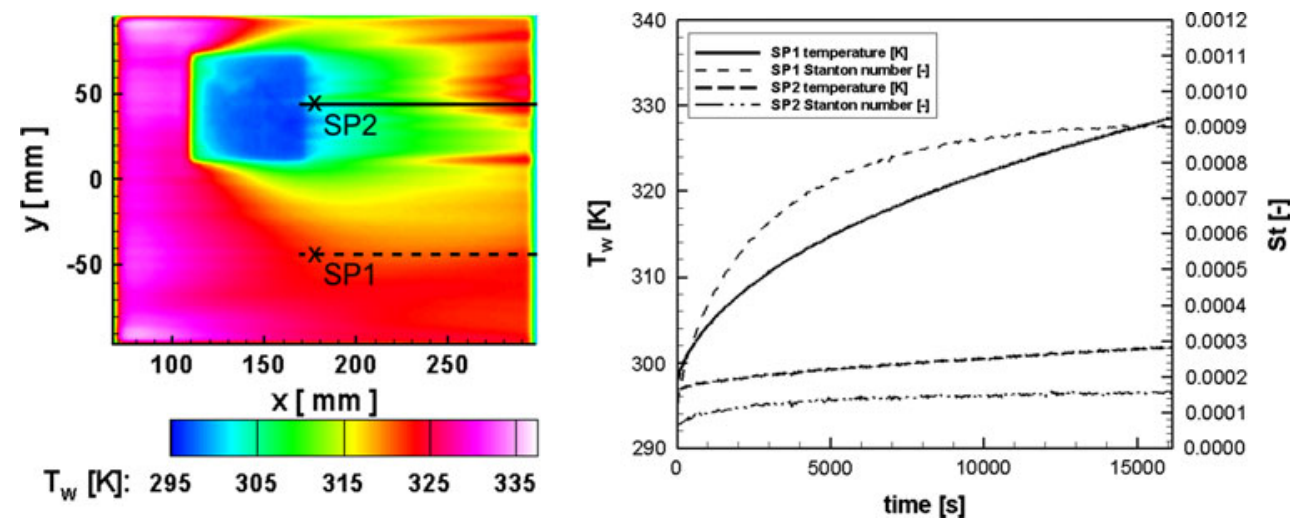

Fig. 6 Measured surface temperature distribution and Stanton number profiles for the test at angle of attack of $-5^{\circ}$ and FC 1 with air as coolant for $F=8.48 \times 10^{-3}(\mathbf{a}, \mathbf{b})$ and $F=16.95 \times 10^{-3}(\mathbf{c}, \mathbf{d})$
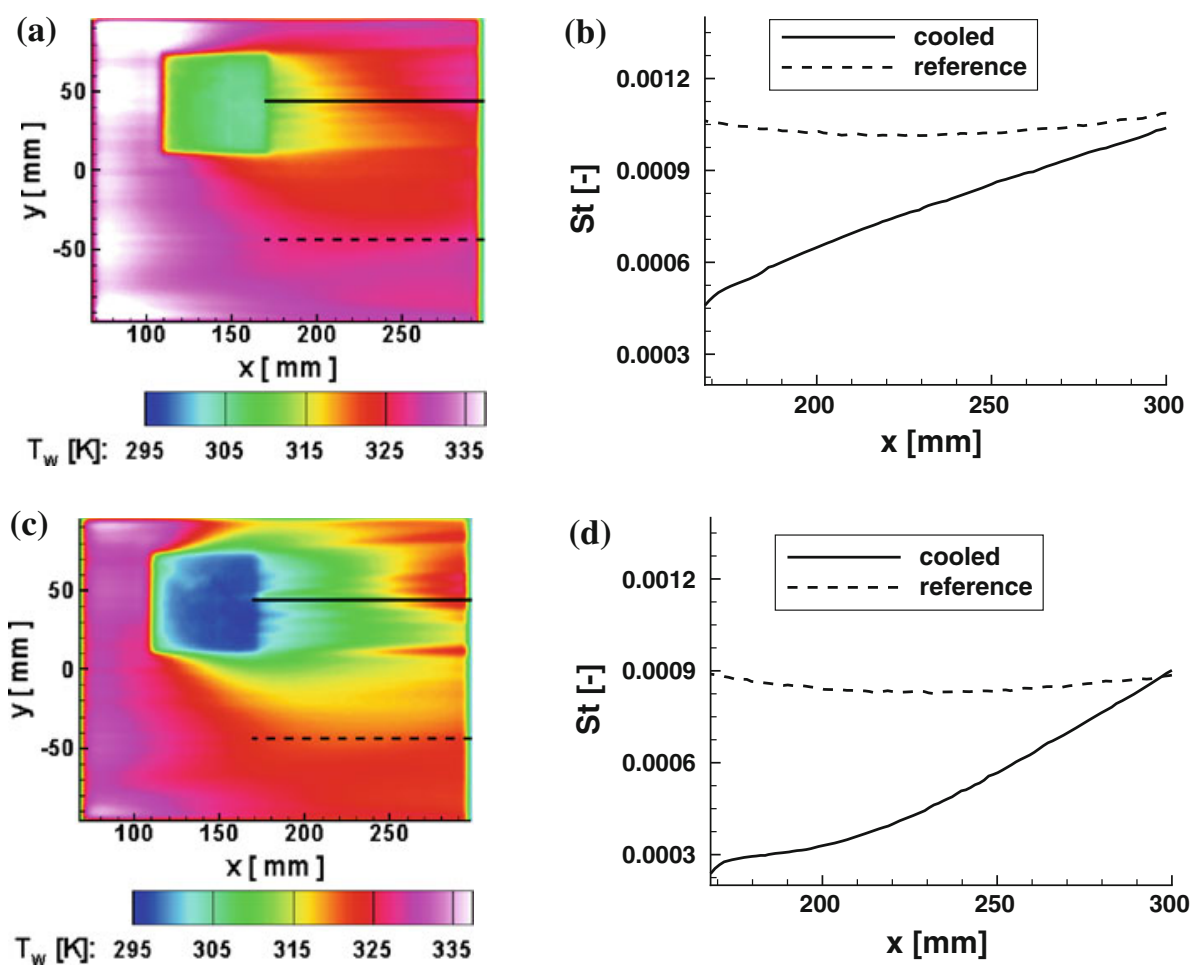

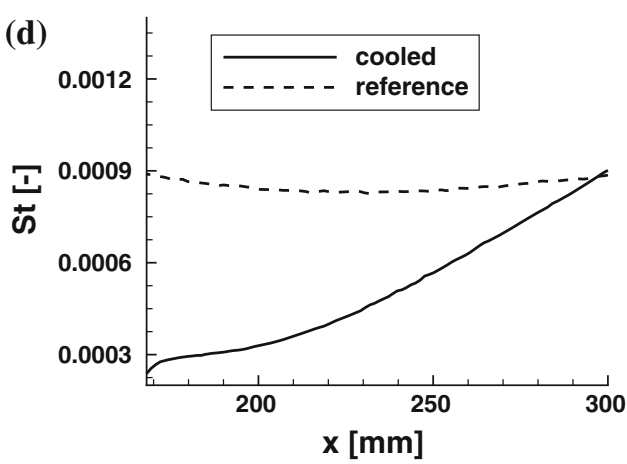

reference part of the model. The data reduction is carried out for the emissivity value of PEEK (0.95). The emissivity of the metallic surface is lower. Therefore, the temperature of the metallic sample also has to be corrected. But, because of its high thermal conductivity and complex internal heat transfer of the metallic probe, the semi-infinite wall assumption is not valid. Therefore, the heat flux determination has not been carried out for the porous part of the model.

Increased blowing ratio leads to a stronger cooling downstream of the porous sample and covers a broader surface in perpendicular direction. However, toward the downstream end of the model, the aerothermal heating for both cases becomes comparable. This is correlated to some local turbulent or vortex structures induced by the interaction of the coolant gas with the supersonic cross flow at high blowing ratios (Gülhan et al. 2008). IR images show that the coolant flow through the porous surface is not homogenous and causes slight heterogeneous flow topology like transition strips downstream. The cooling efficiency of both tests is shown in Fig. 7. It is quite high (above 0.6) on the porous sample itself and just downstream of it becomes weaker along the flow axis on the surface. Here, as mentioned before, the data on the sample surface has to be considered qualitatively, since the semiinfinite wall assumption is not valid. The development of the temperature and heat flux rate on the surface of the reference model part indicates that in the downstream part of the model the flow is not fully laminar any more. The negative efficiency value just upstream of the porous sample is related to the heat transfer from the PEEK plate to the metallic cooled porous sample. Such lateral heat 


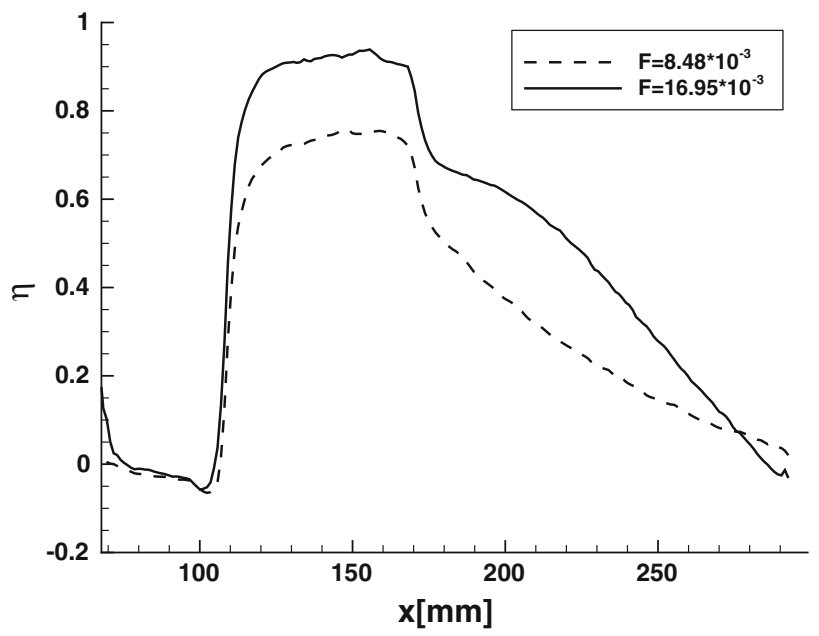

Fig. 7 Calculated cooling efficiency for the test at angle of attack of $-5^{\circ}$ and FC 1 with air as coolant

fluxes are not captured with a one-dimensional model, which has been used in this study.

Tests with argon as a coolant provide similar results as air (Fig. 8). The only remarkable difference is slightly weaker hot spots around the coolant film. Stronger interaction between the coolant flow and cross flow of argon compared to air could be the main reason of this discrepancy.

In contrast to the argon case, tests with helium provide significant differences compared to the data of tests with air as coolant. At the same mass flow rate, the volume rate of helium is factor seven and ten higher than air and argon, respectively. In addition, the heat capacity and thermal conductivity of helium are larger. Even at the low blowing ratio $\left(F=8.48 \times 10^{-3}\right)$, i.e. at coolant mass flow rate of $0.2 \mathrm{~g} / \mathrm{s}$, helium causes stronger cooling compared to air and argon at high blowing ratio (Fig. 9). Because of 3D effects the cooled region also expands perpendicular to the flow direction.

Doubling the helium mass flow rate to $0.4 \mathrm{~g} / \mathrm{s}$, i.e. $F=16.95 \times 10^{-3}$ causes the coolant film remains stable until the downstream end of the model (Fig. 9c). At this condition, the coolant gas influences the cross flow strongly and causes effects like a side jet in supersonic flows. Shear layer and horse shoe vortexes form and induce high local aerothermal heating in the edge areas of the coolant flow. In contrast to air as Schlieren pictures (see Fig. 10) show, a stronger shock forms ahead of the helium coolant flow and it induces flow separation and re-attachment at the leading edge of the porous sample.

Figure 11 shows a comparison of the efficiency of different coolants at two different blowing ratios. As mentioned before, the cooling efficiency in the range of the metallic porous sample has to be interpreted only qualitatively. However, the data on the PEEK model surface show clearly that air and argon as coolant provide similar cooling efficiency. At low blowing ratios, helium cooling allows for a factor two better cooling efficiency compared to other
Fig. 8 Measured surface temperature distribution and Stanton number profiles for the test at angle of attack of $-5^{\circ}$ and FC 1 with argon as coolant for $F=8.48 \times 10^{-3}(\mathbf{a}, \mathbf{b})$ and $F=16.95 \times 10^{-3}(\mathbf{c}, \mathbf{d})$
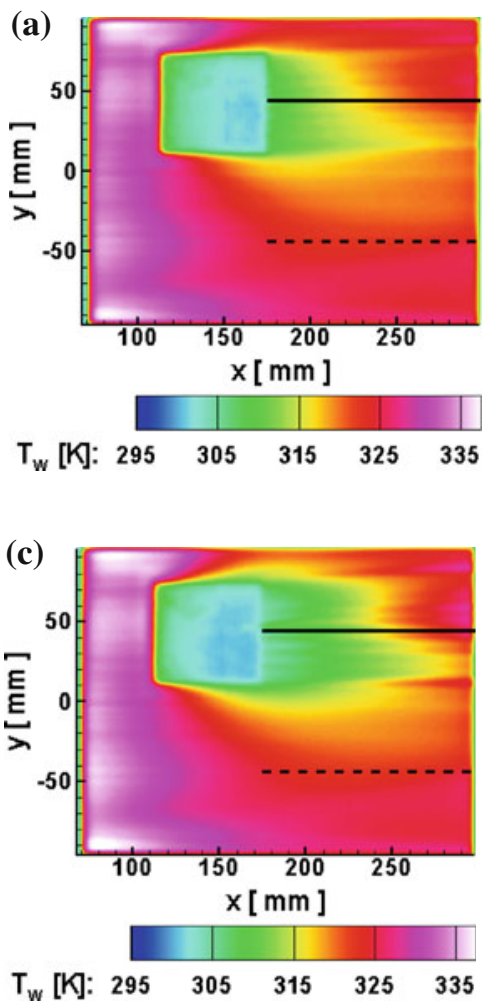
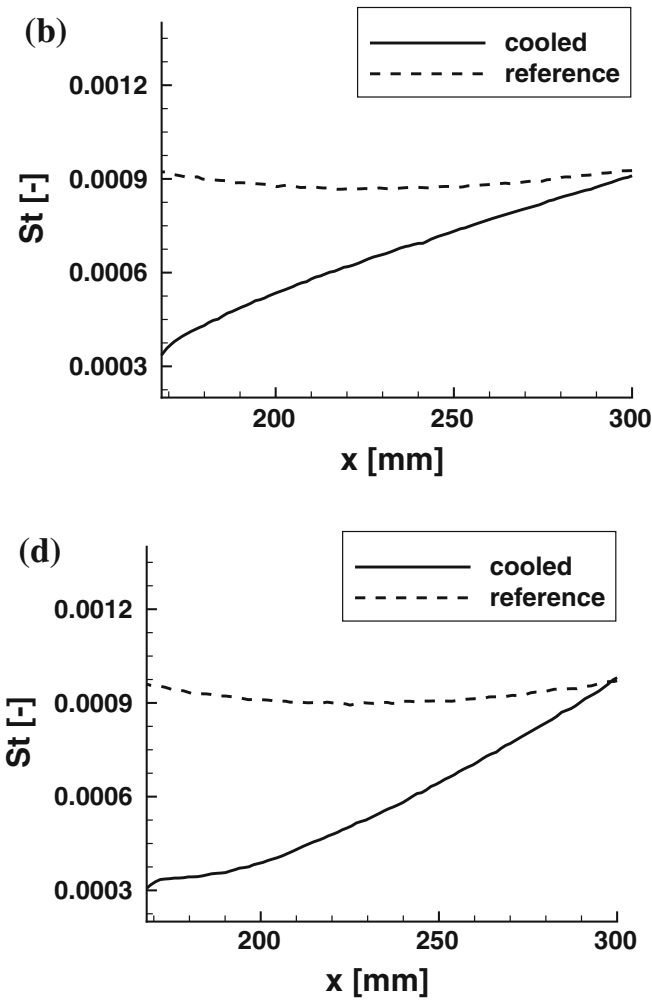
Fig. 9 Measured temperature distribution and Stanton number profiles for the test at angle of attack of $-5^{\circ}$ and FC 1 with helium as coolant for $F=8.48 \times 10^{-3}(\mathbf{a}, \mathbf{b})$ and $F=16.95 \times 10^{-3}(\mathbf{c}, \mathbf{d})$
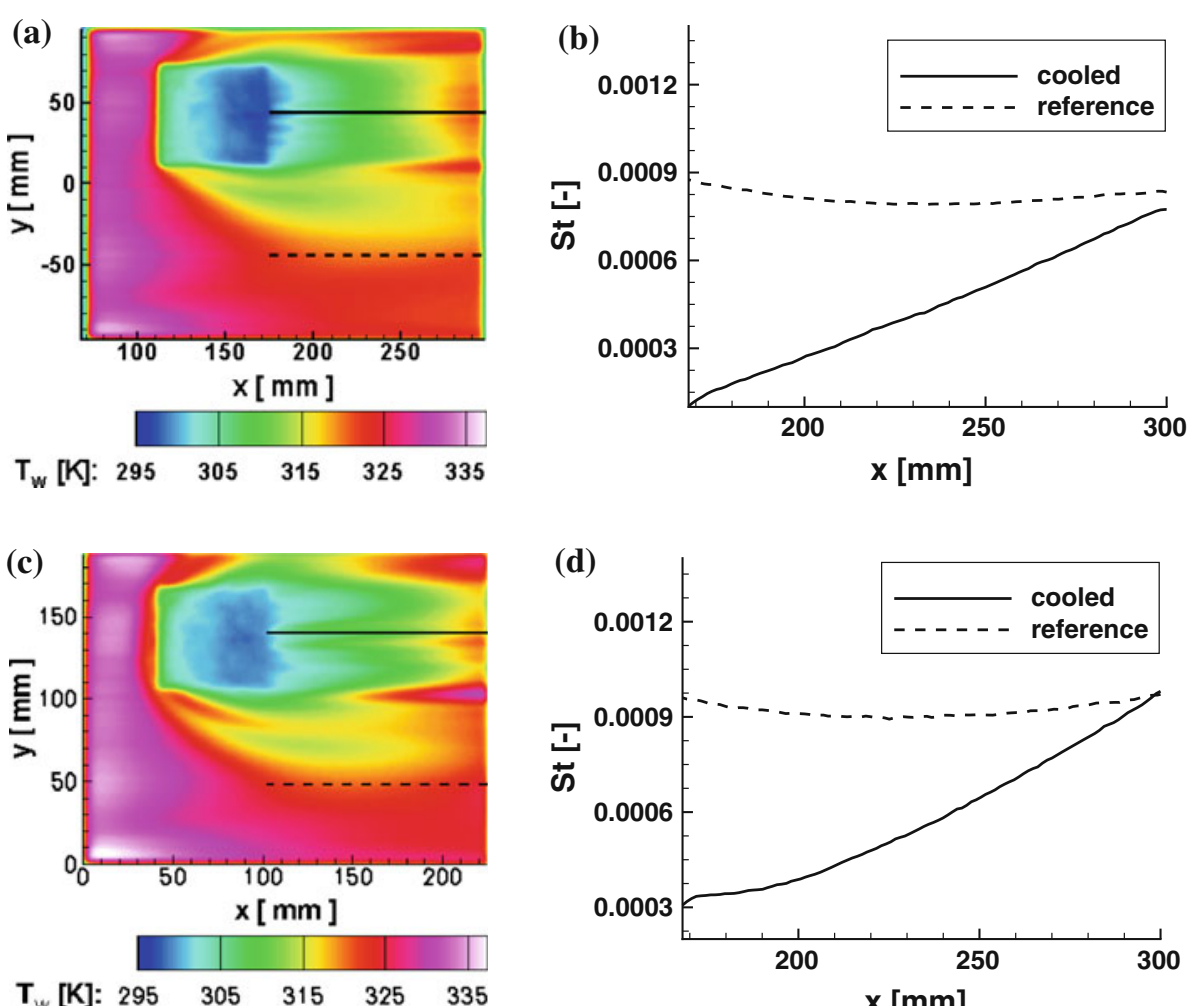

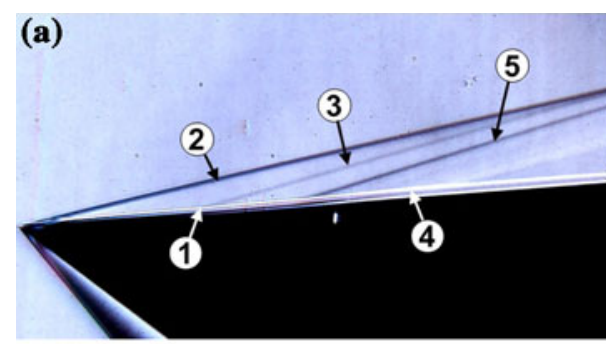

(b)

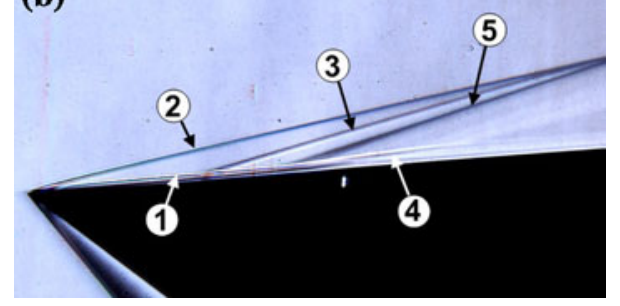

Fig. 10 Schlieren pictures of tests at angle of attack of $-5^{\circ}$ and FC 1 with air (a) and helium (b) as coolant and blowing ratio of $F=15.48 \times 10^{-3}$ : (1) boundary layer, (2) leading edge shock, (3) weak interface shock, (4) coolant film, (5) separation shock

two gases. Just around the downstream edge of the plate, the helium film breaks down gradually and becomes less efficient. In case of high blowing ratio, we observe a different development. Here compared to the low blowing ratio case, the cooling efficiency of the helium on the porous sample itself is a little bit lower, but the film is efficient even at the downstream edge of the model. A further interesting aspect is the slight structure cooling upstream of the porous sample. It is related to the flow separation and suctioning of the coolant gas to the surface by separated flow behind the separation shock ahead of the sample. We should keep in mind that local hot spots resulting in shear layers, vortexes and flow re-attachment downstream could damage the structure. Therefore, the high blowing ratios in laminar flows seem to be a problem.

In order to study the active cooling efficiency in turbulent flows, the unit Reynolds number is increased more than factor 5 to $4.56 \times 10^{6}$. As shown in Fig. 12, the boundary layer transition takes place first around the middle of the test model. Therefore, only one test with $0.2 \mathrm{~g} / \mathrm{s}$ argon cooling is performed. Since the heat increase induced by natural transition is one of the most important problems of spacecrafts, the data of this test is presented here. It is interesting to see that the active cooling at the blowing ratio of $3.02 \times 10^{-3}$ (blowing ratio change comes from different cross-flow parameters) is not very efficient in a turbulent flow.

\subsection{Heat flux measurements at angle of attack of $-20^{\circ}$}

An increase of the angle of attack of the plate from $-5^{\circ}$ to $-20^{\circ}$ leads to a heat flux increase of about $35 \%$. The numerical estimation of this configuration at FC I and coolant mass flow rate of $0.4 \mathrm{~g} / \mathrm{s}$ provides a blowing ratio of $F=5.84 \times 10^{-3}$. Again here no remarkable difference 

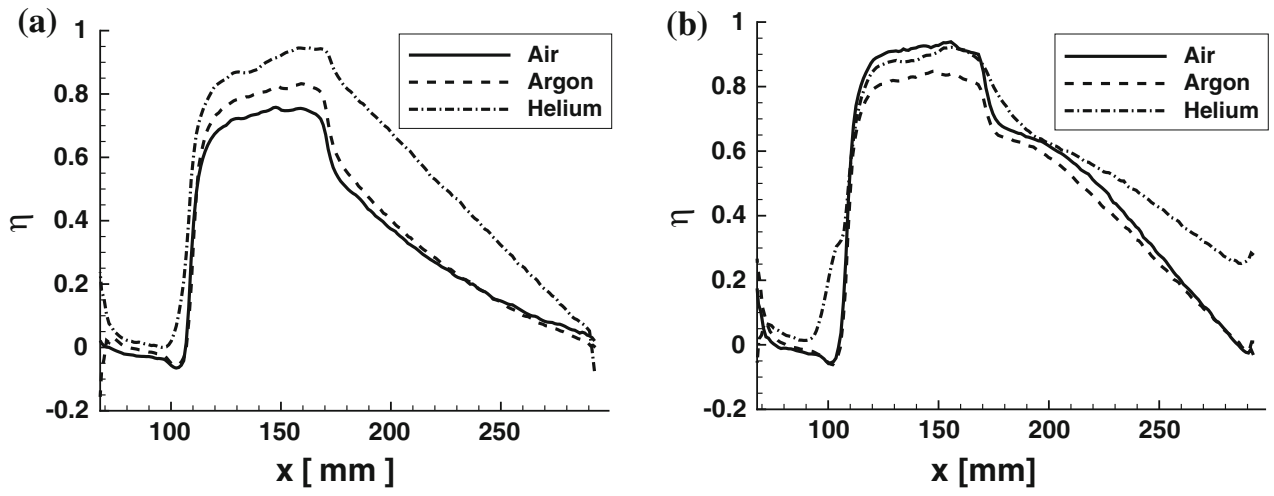

Fig. 11 Comparison of cooling efficiency of different gases at an angle of attack of $-5^{\circ}$ and FC 1, blowing ratio: $F=8.48 \times 10^{-3}(\mathbf{a})$; $F=16.95 \times 10^{-3}(\mathbf{b})$

Fig. 12 Measured temperature distribution and Stanton number profiles for the test at angle of attack of $-5^{\circ}$ and FC II with argon as coolant for $F=3.02 \times 10^{-3}$
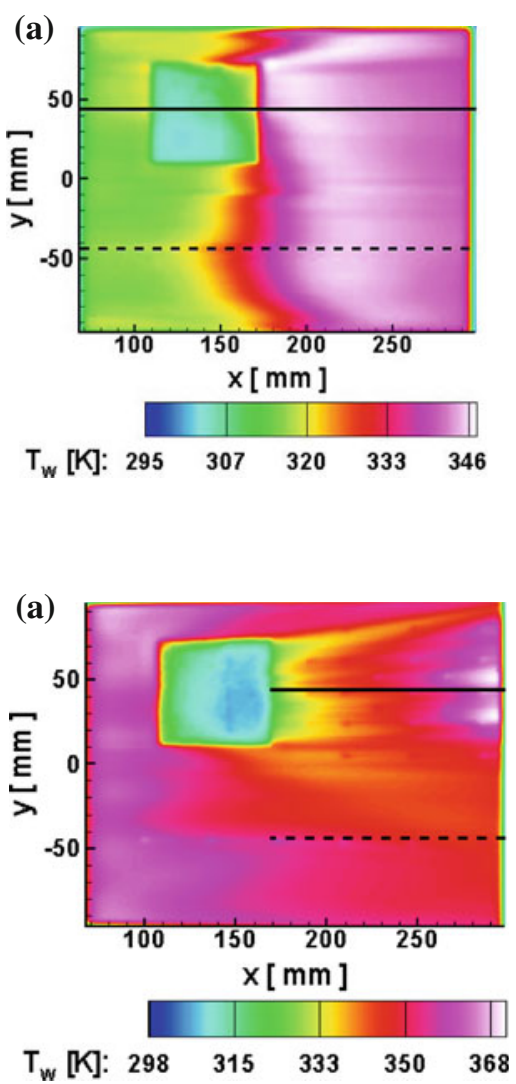

Fig. 13 Measured surface temperature distribution and Stanton number profiles for the test at angle of attack of $-20^{\circ}$ and FC I with air (a, b) and argon (c, d) as coolant for $F=5.84 \times 10^{-3}$
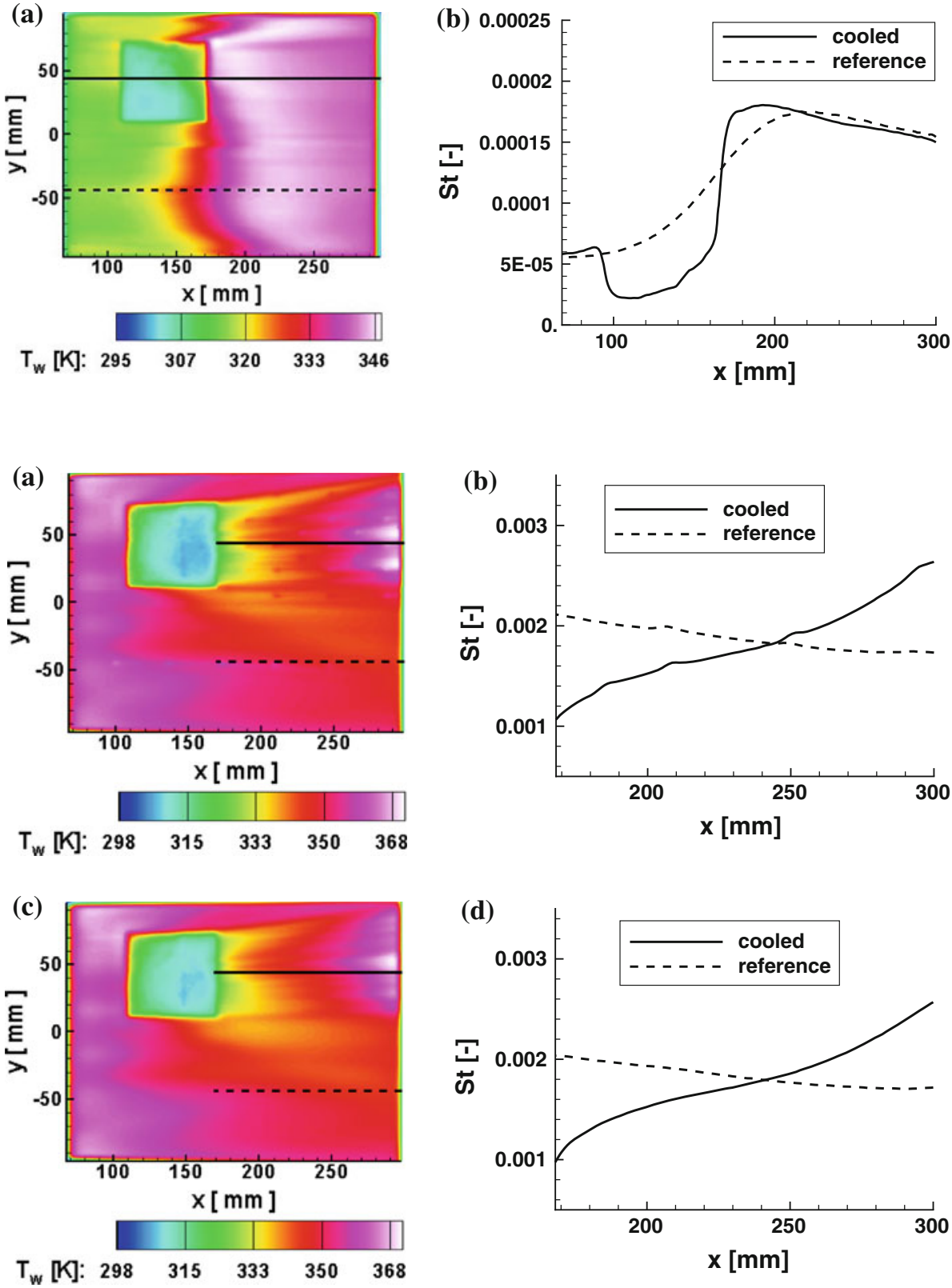
Fig. 14 Measured temperature distribution and Stanton number profiles for the test at angle of attack of $-20^{\circ}$ and FC I with helium as coolant for $F=5.84 \times 10^{-3}$
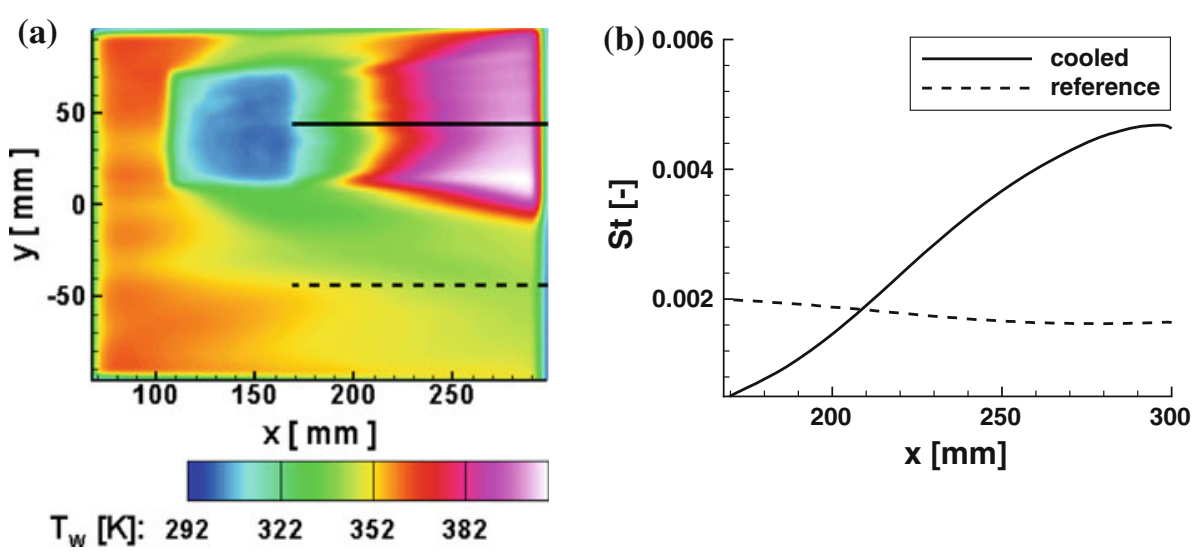

Fig. 15 Measured surface temperature distribution and Stanton number profiles for the test at angle of attack of $-20^{\circ}$ and FC II $\left(F=1.38 \times 10^{-3}\right)$. Coolant: air (a, b); $\operatorname{argon}(\mathbf{c}, \mathbf{d})$; helium $(\mathbf{e}, \mathbf{f})$
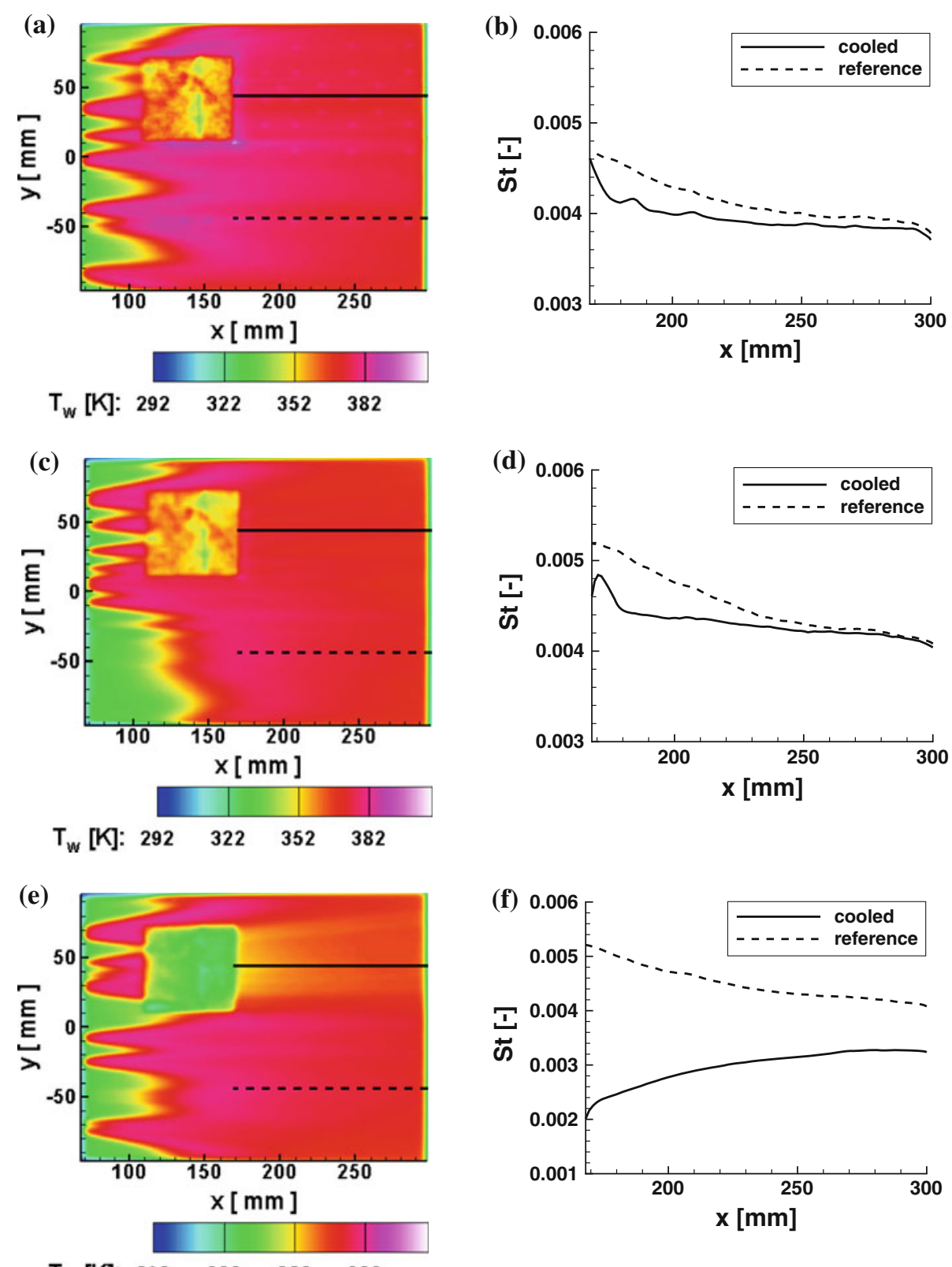

$\mathrm{T}_{\mathrm{w}}[\mathrm{K}]: \begin{array}{llll}292 & 322 & 352 & 382\end{array}$ 
in the temperature distribution of tests with air and argon could be identified (Fig. 13). The region up to $60 \mathrm{~mm}$ downstream of the porous sample is affected by cooling gas. In the further downstream region, higher heating compared to the reference surface is occured. It is caused by the interference of the cooling film with the external flow. Again here vortex formation and corresponding boundary layer tripping could be the main reason of this phenomenon.

At an angle of attack of $-20^{\circ}$ using helium as cooling medium, the cooled region behind the porous sample becomes smaller (Fig. 14). The high volume flow of helium leads to boundary layer transition and a strong increase in aerothermal heating.
Increasing the Reynolds number from $0.73 \times 10^{6}$ to $4.56 \times 10^{6}$ causes a boundary layer transition around the upstream edge of the PEEK plate. The blowing ratio at $0.4 \mathrm{~g} / \mathrm{s}$ mass flow rate is now $F=1.38 \times 10^{-3}$. As shown in Fig. 15, air and argon induce only a weak cooling effect at this blowing rate. Since the boundary layer transition in the upstream part of the PEEK plate has also some differences, the small differences in the cooling efficiency cannot be explained easily. Using helium leads to a remarkable reduction of the heating of the PEEK plate downstream of the porous sample. About $30 \%$ decrease of the Stanton number has been measured at the downstream end of the PEEK plate. Since on the porous wall a fully turbulent boundary layer flow is established, there is no
Fig. 16 Measured temperature distribution and Stanton number profiles for the test at angle of attack of $-20^{\circ}$ and FC II $\left(F=2.75 \times 10^{-3}\right)$. Coolant: air $(\mathbf{a}, \mathbf{b})$; $\operatorname{argon}(\mathbf{c}, \mathbf{d})$; helium $(\mathbf{e}, \mathbf{f})$
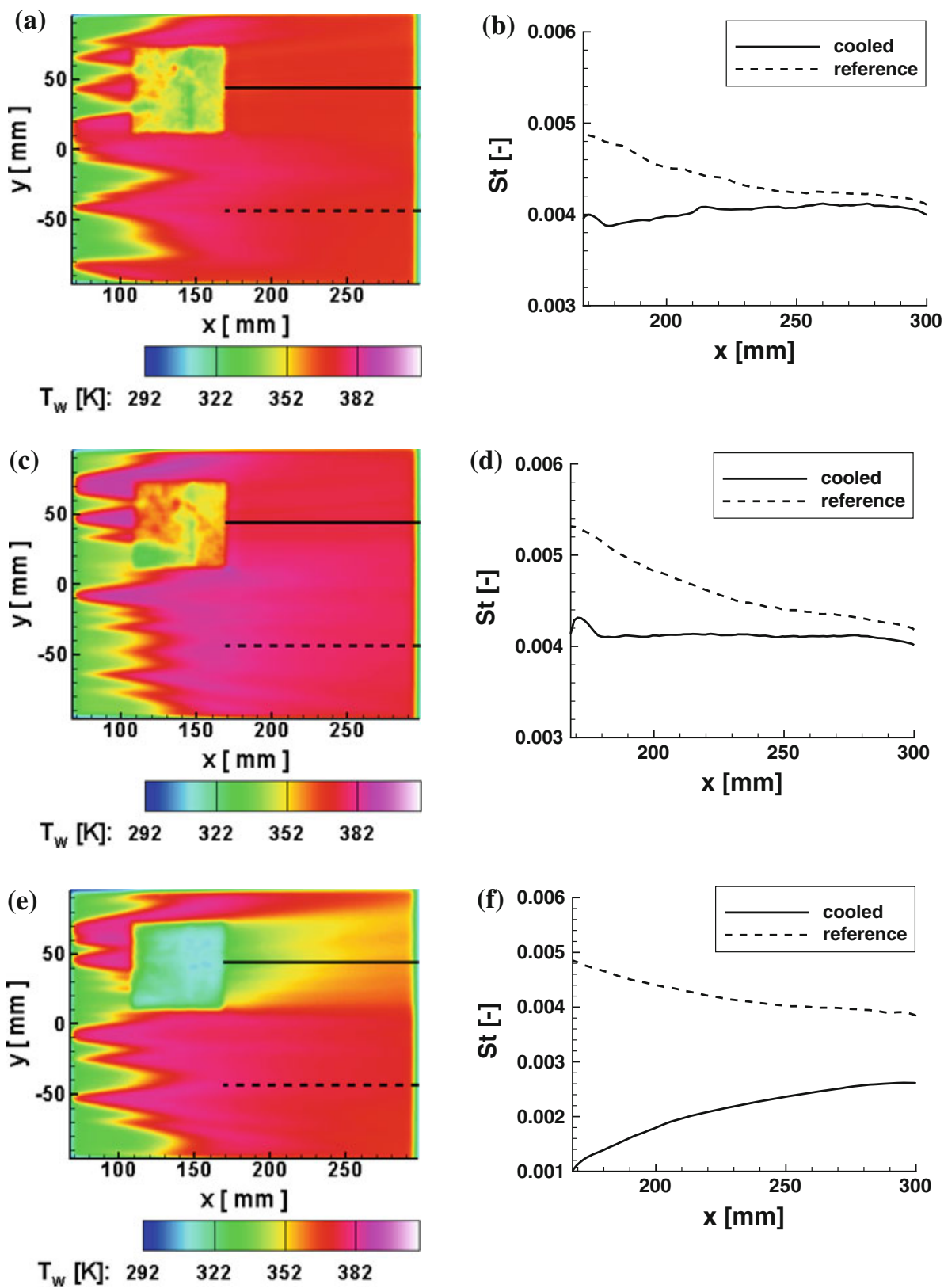


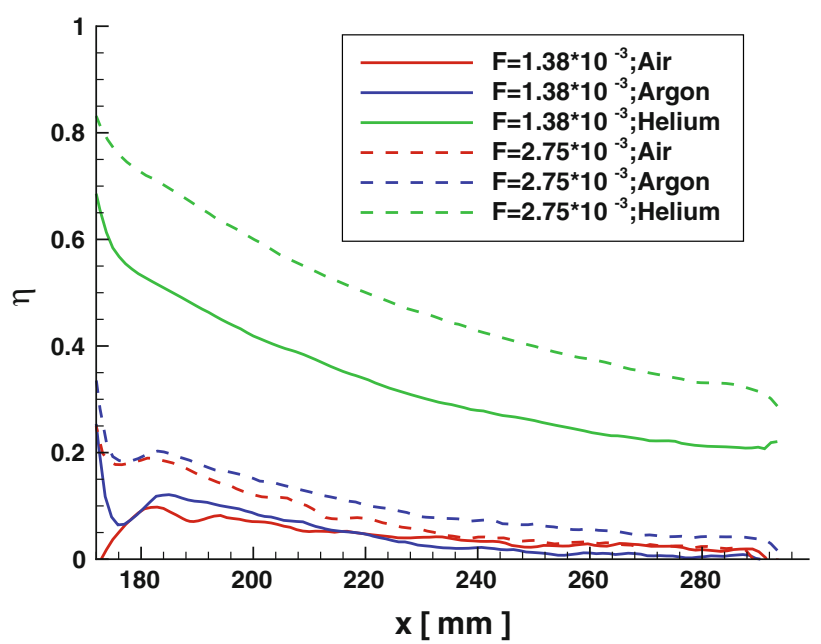

Fig. 17 Cooling efficiency of air, Argon and Helium at angle of attack of $-20^{\circ}$ and FC II for $F=1.38 \times 10^{-3}$ and $F=2.75 \times 10^{-3}$

additional heating coming from the interaction of the cooling flow and cross flow in the downstream region. Therefore, the coolant mass flow rate could be increased without causing hot spots.

Further tests are carried out by doubling the coolant mass flow rate to $0.8 \mathrm{~g} / \mathrm{s}$, i.e. increasing blowing ratio to $F=2.75 \times 10^{-3}$. For air and argon cooling, a Stanton number decrease of about $20 \%$ is achieved immediately behind the porous sample (Fig. 16). In contrast to the $0.4 \mathrm{~g} / \mathrm{s}$ test case, the cooling efficiency of Argon does not change until the downstream end of the plate. Helium shows a significant better cooling efficiency than argon.

The cooling efficiency of all six tests at $-20^{\circ}$ angle of attack is summarized in Fig. 17. As mentioned before, in a fully turbulent flow doubling the coolant mass flow rate, i.e. blowing ratio, increases the cooling efficiency remarkably without inducing any hot spots on the surface. Using air and argon provides the maximum cooling efficiency of less than $20 \%$ even at $0.8 \mathrm{~g} / \mathrm{s}$ mass flow rate. It is significantly lower compared to the laminar flow case. Helium is a very efficient coolant. Even at $0.4 \mathrm{~g} / \mathrm{s}$ helium mass flow rate, a cooling efficiency of $60 \%$ is measured immediately downstream of the porous sample. It decreases along the downstream surface continuously and reaches about $20 \%$ at the end of the PEEK plate. When the helium mass flow rate is increased by factor two, the cooling efficiency reaches a maximum value of $80 \%$ just downstream of the porous sample. It decreases again to $35 \%$ within $120 \mathrm{~mm}$.

\subsection{Pressure measurements at angle of attack of $-5^{\circ}$}

In order to measure the influence of the active cooling on the surface pressure distribution, 28 pressure ports are

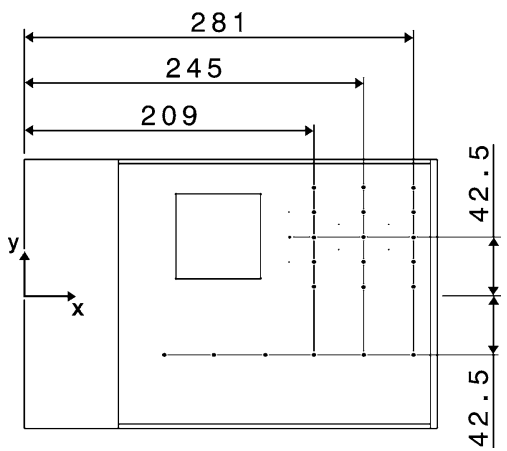

Fig. 18 Pressure ports positions

integrated into the PEEK plate. In the following figures, pressure data measured along two longitudinal rows $(y=42.5 \mathrm{~mm}$ and $y=-42.5 \mathrm{~mm})$ and three perpendicular lines $(x=209 \mathrm{~mm}, x=245 \mathrm{~mm}$ and $x=281 \mathrm{~mm})$ are presented (Fig. 18). The pressure coefficients are defined as the ratio between the measured pressure and dynamic pressure defined of the wind tunnel flow. The accuracy of the pressure coefficient determination is better than $\pm 5 \%$.

The first series of tests is conducted at an angle of attack of $-5^{\circ}$ at low Reynolds number flow conditions (FC I). Using air or argon as coolant, no remarkable changes in the pressure distribution could be measured behind the porous samples. Figure 19 shows the pressure distribution on the surface of the model with air as coolant and two different blowing ratios. All pressure variations are in the range of the calibration accuracy of the pressure transducers, which is $\Delta c_{p}= \pm 0.0019$ or $\pm 17 \mathrm{~Pa}$. For this reason, no statements on the influence of the transpiration cooling on the pressure distribution can be made in this case.

For the case with significant cooling efficiency such as using Helium at high blowing ratio of $F=16.96 \times 10^{-3}$, the influence of the cooling on the pressure is noticeable. Figure 20 shows a comparison of pressure data of the runs with helium and air cooling. Unlike the test with air or argon, high blowing ratios of helium induces a significant pressure reduction behind the porous sample. Results for the pressure distribution are shown in Fig. 20, where data obtained in a test with helium and a blowing ratio of $F=16.96 \times 10^{-3}$ are compared to a test using air as coolant and a blowing ratio of $F=8.48 \times 10^{-3}$. Both along the $x$ axis and $y$ axis pressure changes can be observed. In case of the test with helium, pressure differences of more than $100 \mathrm{~Pa}$ between cooled and non-cooled part of the model are ascertainable.

4.4 Pressure measurements at angle of attack of $-20^{\circ}$

At the angle of attack of $-20^{\circ}$, the pressure distribution shows a similar behavior as the test data at $-5^{\circ}$. As shown in Fig. 21 at blowing ratio of $F=5.84 \times 10^{-3}$ using 

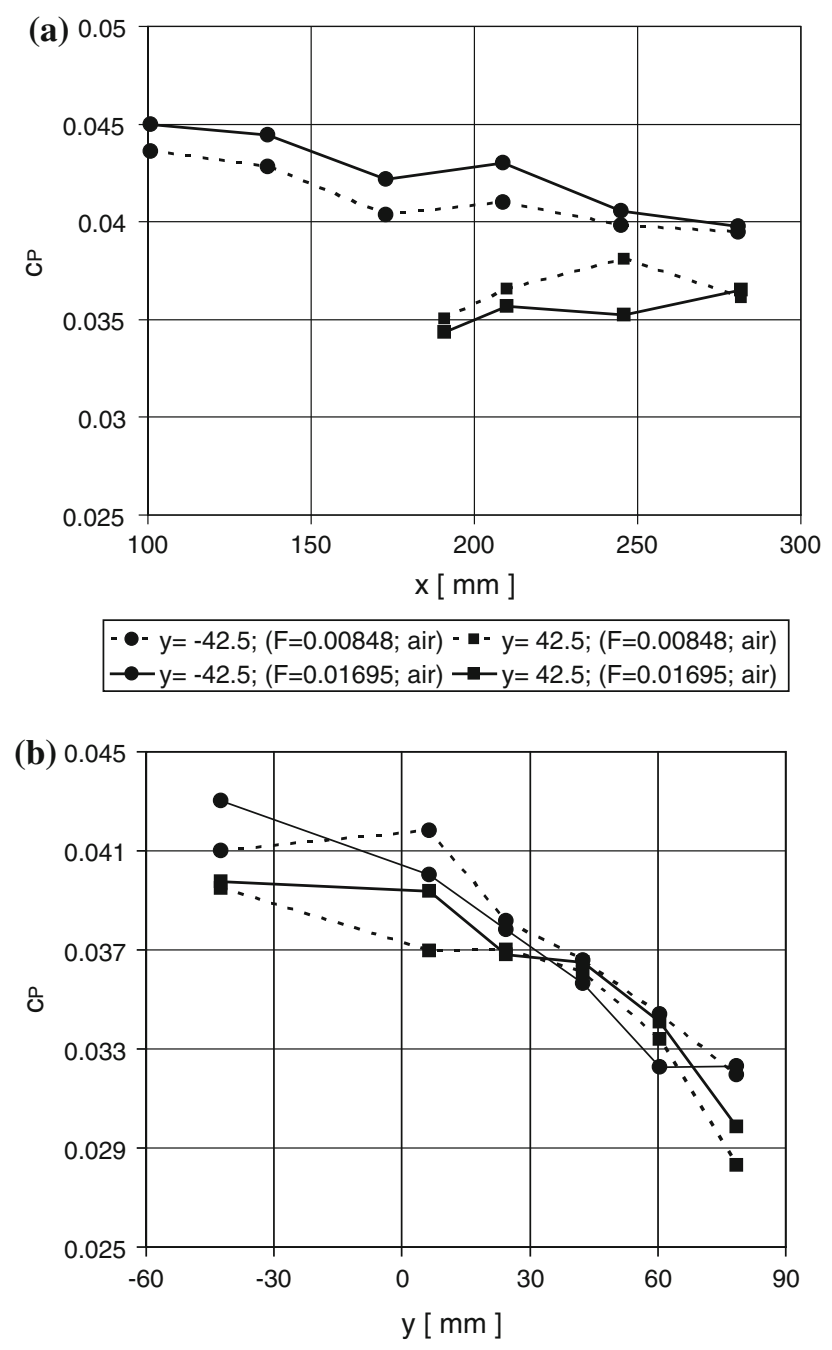

$-\mathrm{x}=209 \mathrm{~mm} ;(\mathrm{F}=0.00848$; air) $-\boldsymbol{\square}-\mathrm{x}=281 \mathrm{~mm} ;(\mathrm{F}=0.00848 ;$ air $)$
$-\mathrm{x}=209 \mathrm{~mm} ;(\mathrm{F}=0.01695 ;$ air $) \rightarrow-x=281 \mathrm{~mm} ;(\mathrm{F}=0.01695 ;$ air $)$

Fig. 19 Pressure distributions at angle of attack of $-5^{\circ}$ and FC I with air as coolant

$0.4 \mathrm{~g} / \mathrm{s}$ argon as cooling medium the pressure downstream of the porous sample decreases slightly and increases again to the same level as the reference surface around the downstream end of the model surface. Compared to argon, helium leads to remarkable decrease of the pressure distribution.

Tests conducted at an angle of attack of $-20^{\circ}$ at low Reynolds numbers showed similar results as the tests at an angle of attack of $-5^{\circ}$. Results for a test with a blowing ratio of $F=5.48 \times 10^{-3}$ using $0.4 \mathrm{~g} / \mathrm{s}$ argon as cooling medium are given in Fig. 21. The pressure distribution is compared to the data gained from a test without cooling. Directly behind the porous sample $(x=209 \mathrm{~mm})$ pressure slightly decreases, whereas near the trailing edge of the model surface $(x=281 \mathrm{~mm})$, no essential differences between the two tests are measurable.

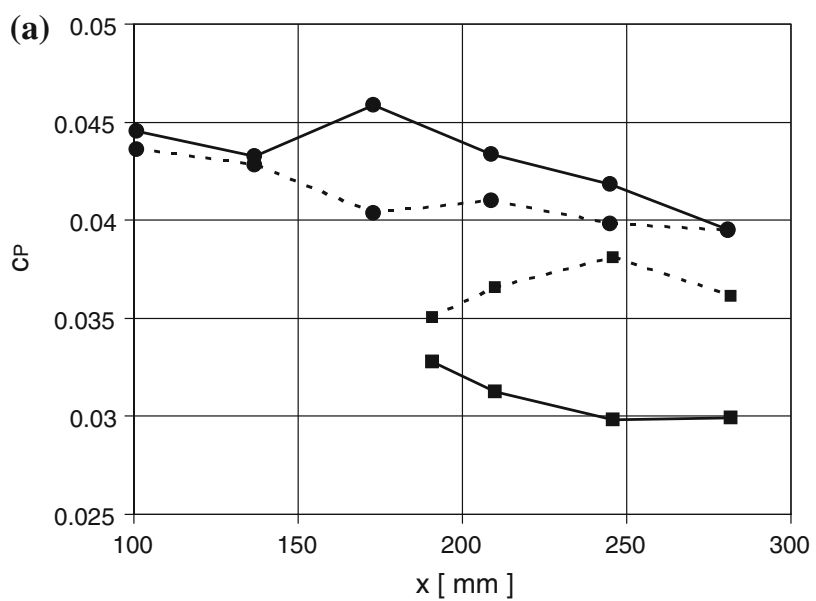

- $y=-42.5 ;(F=0.00848$; air) - $-\mathrm{y}=42.5 ;(\mathrm{F}=0.00848$; air $)$ $\rightarrow y=-42.5 ;(F=0.01695 ; \mathrm{He}) \rightarrow-y=42.5 ;(F=0.01695 ; \mathrm{He})$

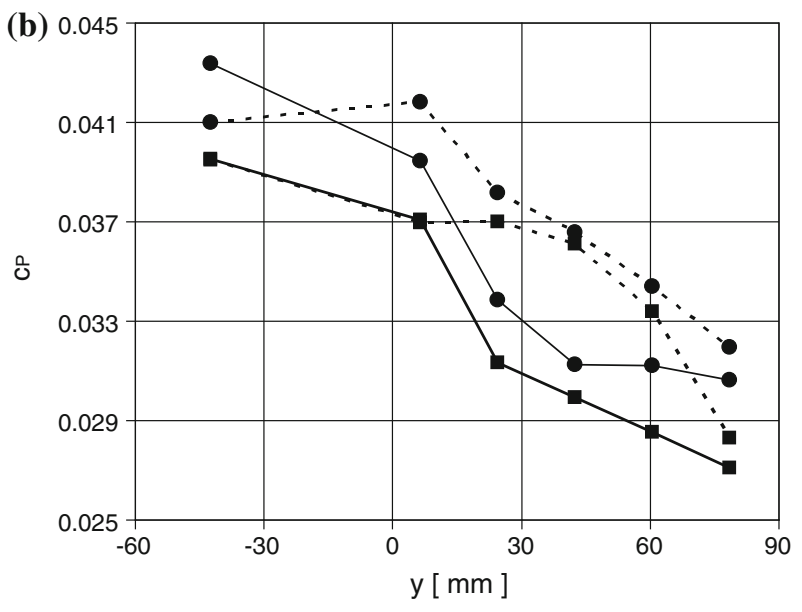

- $\mathrm{x}=209 \mathrm{~mm} ;(\mathrm{F}=0.00848$; air) - $\mathrm{a}-\mathrm{x}=281 \mathrm{~mm} ;(\mathrm{F}=0.00848$; air $)$ - $x=209 \mathrm{~mm} ;(\mathrm{F}=0.01695 ; \mathrm{He}) \rightarrow-x=281 \mathrm{~mm} ;(\mathrm{F}=0.01695 ; \mathrm{He})$

Fig. 20 Pressure distribution at angle of attack of $-5^{\circ}$ with blowing ratios of $F=8.48 \times 10^{-3}$ (air) and $F=16.95 \times 10^{-3}$ (Helium)

In contrast to the argon case, helium leads to remarkable reduction in the static pressure (Fig. 22). Pressure reductions of 950 and $500 \mathrm{~Pa}$ are measured at the first and second pressure port locations downstream of the porous sample, respectively.

In order to check the influence of the temperature deviation on the pressure distribution, both temperature and pressure distributions are given in Fig. 23. It is obvious that the changes in the pressure distribution are not caused only by the temperature change. Although the surface temperatures at the second pressure port downstream of the porous sample $(x=209 \mathrm{~mm})$ for both the reference test without cooling and test with helium cooling are the same, the pressure difference is $c_{p}=0.02(=179 \mathrm{~Pa})$. Even the temperature difference of about $60 \mathrm{~K}$ at the trailing edge of the model does not show any remarkable influence on the pressure distribution. It has to be mentioned here that 

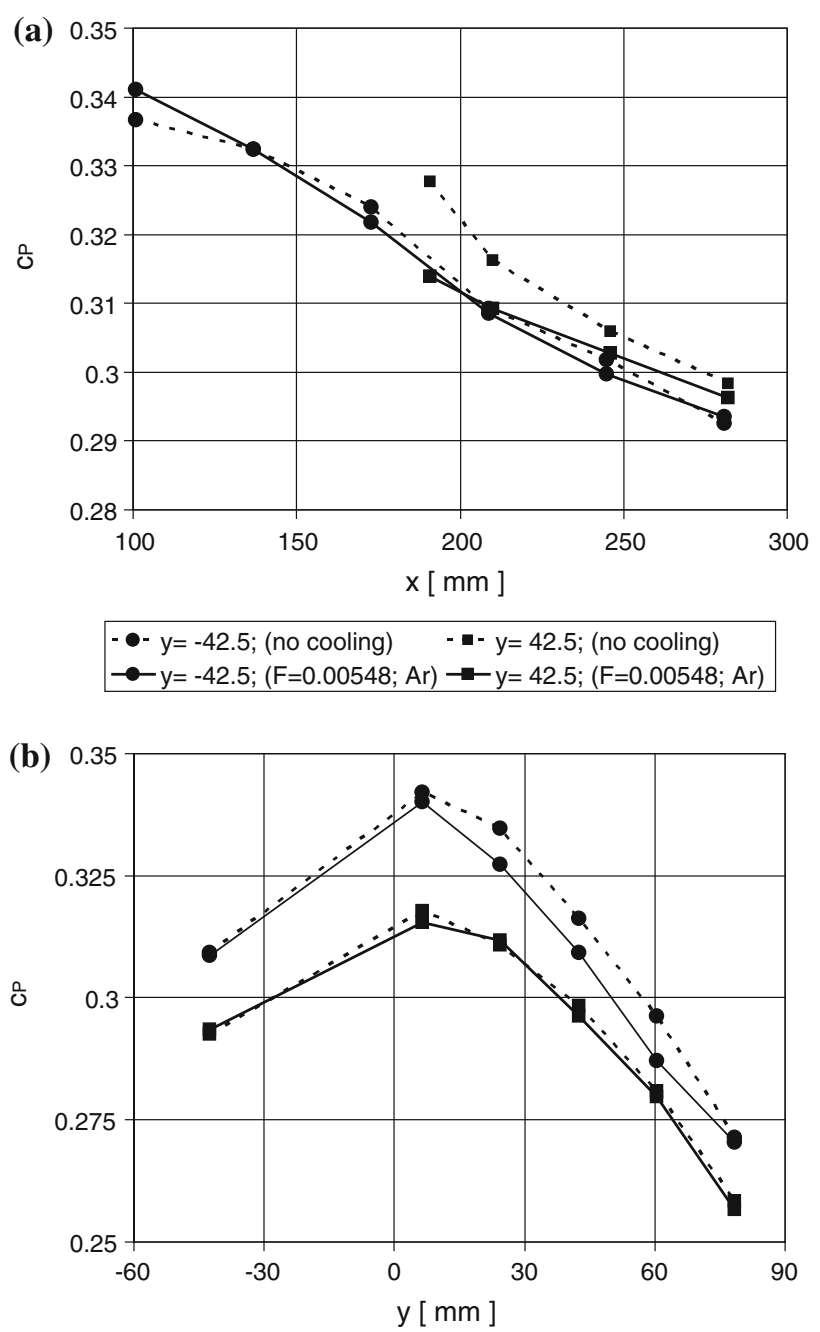

- $\mathrm{x}=209 \mathrm{~mm}$; (no cooling) - $\mathrm{a}-\mathrm{x}=281 \mathrm{~mm}$; (no cooling)

$-x=209 \mathrm{~mm} ;(\mathrm{F}=0.00548 ; \mathrm{Ar}) \rightarrow \mathrm{x}=281 \mathrm{~mm} ;(\mathrm{F}=0.00548 ; \mathrm{Ar})$

Fig. 21 Pressure distribution at angle of attack of $-20^{\circ}$ and FC I

during the run with helium the boundary layer downstream of the porous sample becomes transitional and causes an increase in particular in the surface temperature (see Fig. 14).

During tests at FC II (high Reynolds number) and an angle of attack of $-20^{\circ}$, a similar behavior of the pressure distribution has been observed. Similar to heat flux data a noticeable influence could only be measured at high coolant mass flow rates. Pressure data gained from a test with a mass flow rate of $0.8 \mathrm{~g} / \mathrm{s}$ argon $\left(F=2.75 \times 10^{-3}\right)$ are shown in Fig. 24. Even at such high mass flow rates of argon or air, the pressure change is negligible. Once again tests with helium as cooling gas show some effects. A helium mass flow rate of $0.8 \mathrm{~g} / \mathrm{s}\left(F=2.75 \times 10^{-3}\right)$ yields to a pressure decrease even at the second row of pressure ports $(x=245 \mathrm{~mm})$ downstream of the porous sample (Fig. 25).
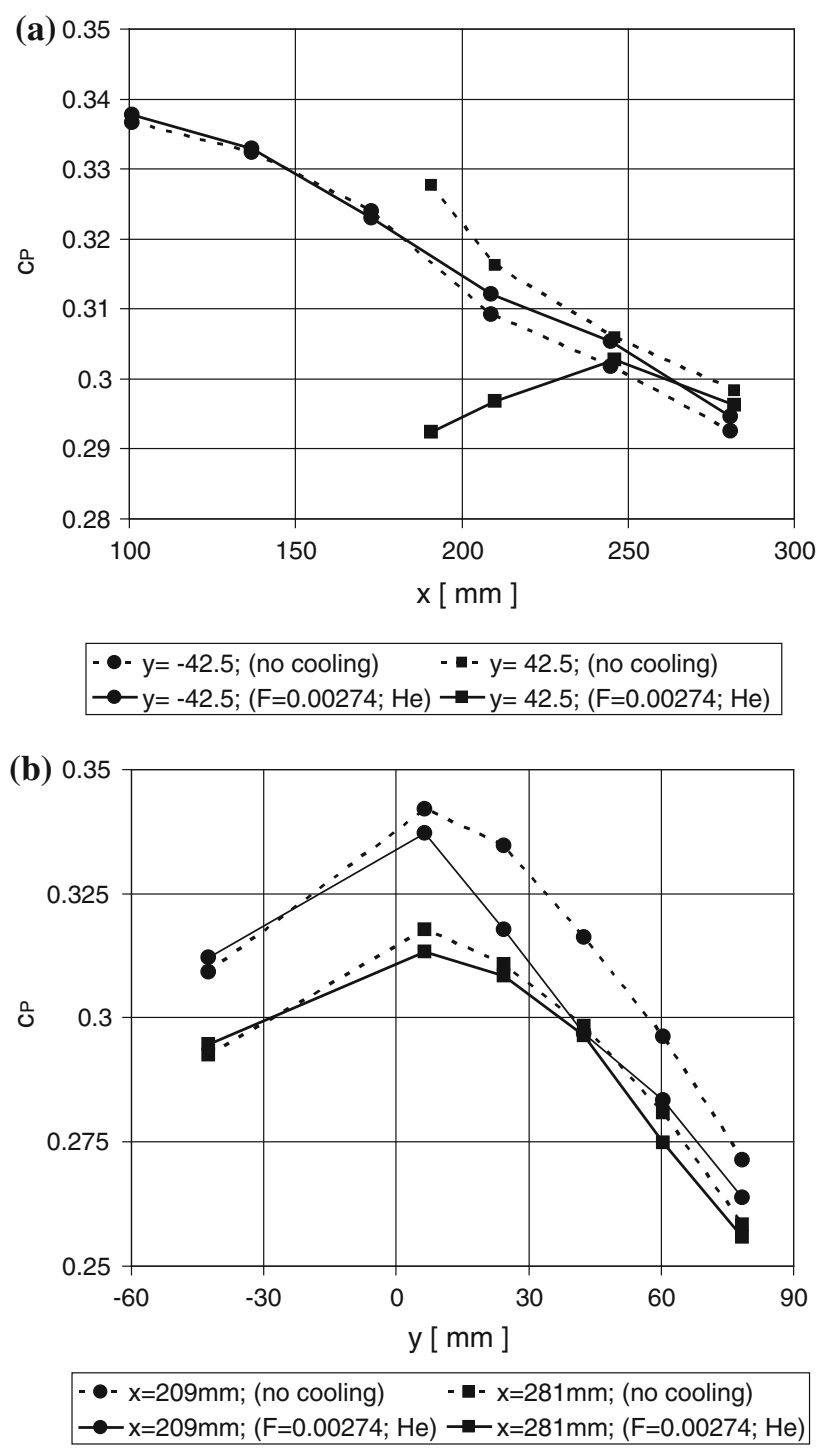

Fig. 22 Pressure distribution at angle of attack of $-20^{\circ}$ and FC I

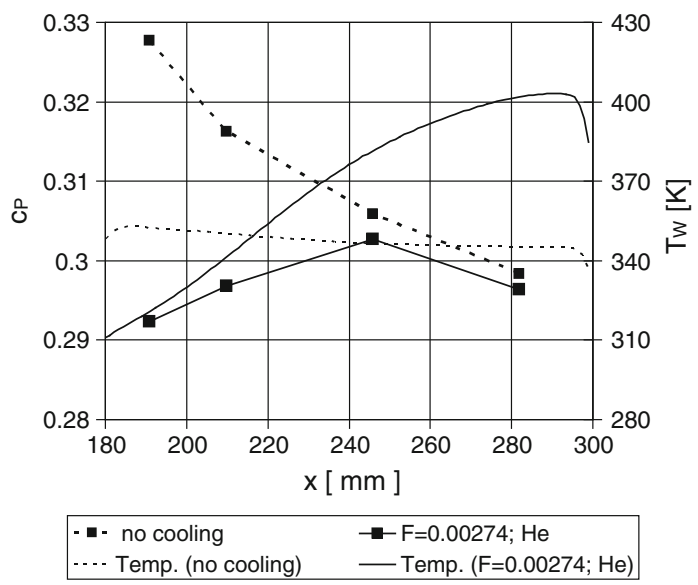

Fig. 23 Measured pressure and surface temperature profiles without and with transpiration cooling (helium as coolant) at angle of attack of $-20^{\circ}$ and FC II $(y=42.5 \mathrm{~mm})$ 

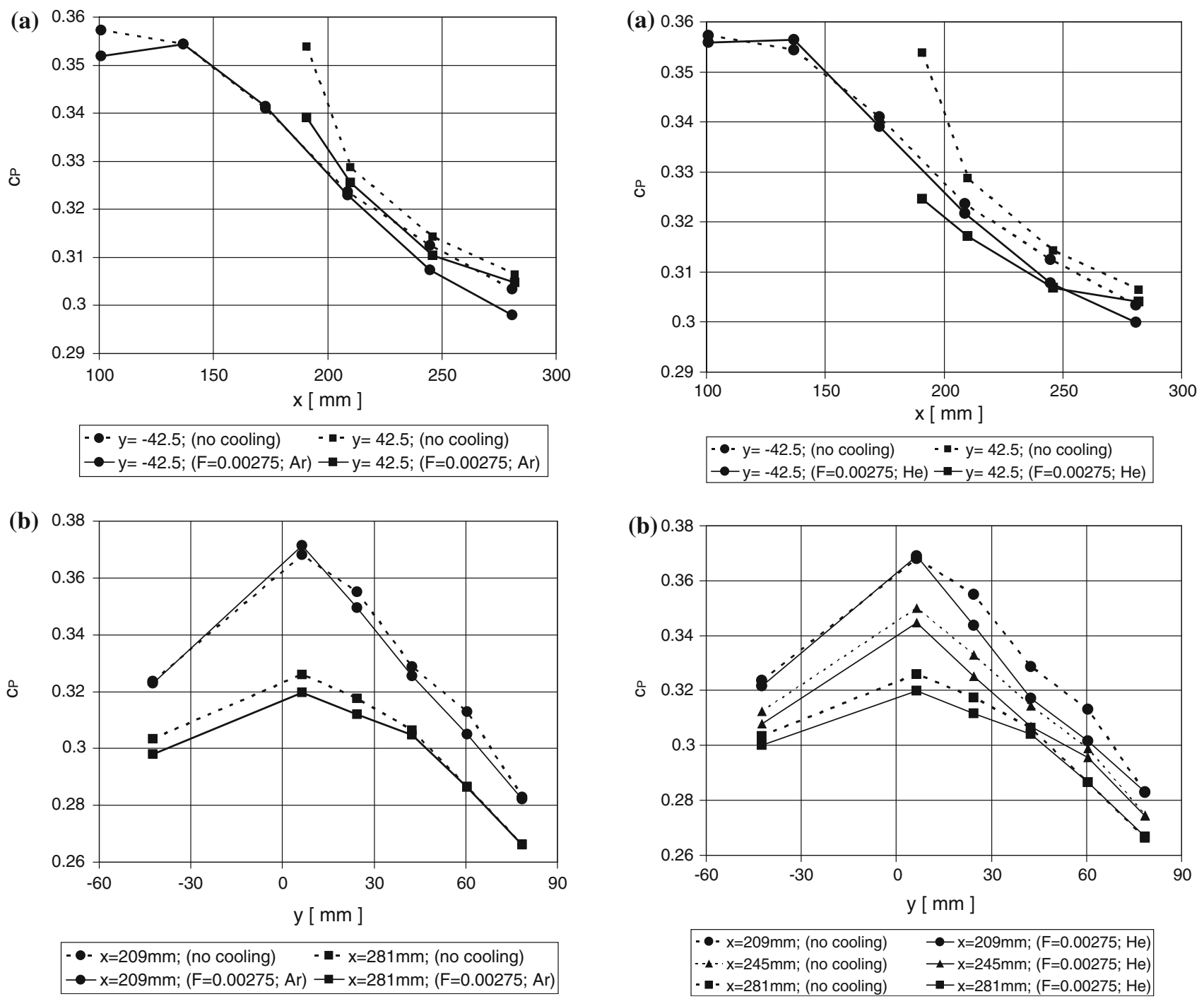

Fig. 24 Measured axial (a) and lateral (b) pressure profiles without and with transpiration cooling (argon as coolant) at angle of attack of $-20^{\circ}$ and FC II

\section{Conclusions}

In the present study, dedicated experiments have been carried out with respect to the influence of the boundary layer state of the hypersonic cross flow on the transpiration cooling efficiency. Experiments were carried out in the DLR hypersonic wind tunnel $\mathrm{H} 2 \mathrm{~K}$ in Cologne at Mach 6 by varying the Reynolds number. In order to demonstrate the efficiency of coolant, air, argon and helium are compared.

At low Reynolds number of $0.73 \times 10^{6}$, laminar flow regime, the high cooling efficiency above 0.6 is measured on the surface of the model just downstream of the porous sample. The cooling efficiency decreases in terms of the distance from the downstream edge of the sample. The sufficient cooling efficiency can be achieved into a broader area by increasing the mass flow rate of a coolant.

Fig. 25 Measured axial (a) and lateral (b) pressure profiles without and with transpiration cooling (helium as coolant) at angle of attack of $-20^{\circ}$ and $\mathrm{FC}$ II

However, the high mass flow rate introduces local hot spots because of boundary layer transition and vortex flows. The compared results show air and argon gives equivalent cooling efficiency. Helium gives significantly better cooling performance at the same mass flow rate, i.e. blowing ratio. At the coolant mass flow rate of $0.4 \mathrm{~g} / \mathrm{s}$, blowing ratio of $5.84 \times 10^{-3}$, air and argon give comparable cooling efficiency in the region up to $60 \mathrm{~mm}$ downstream of the porous sample. In the further downstream area, the interference of the cooling film by the external flow gives higher heating compared to the reference surface, because of the vortex formation and corresponding local boundary layer transition. Compared to air, helium provides a smaller cooling region behind the porous sample and it introduces higher increase of aerothermal heating due to the boundary layer transition. 
At a fully established turbulence, boundary layer flow over the complete model surface which can be achieved by increasing the Reynolds number from $0.73 \times 10^{6}$ to $4.56 \times 10^{6}$ at $20^{\circ}$ angle of attach, air and argon provide a significantly weaker cooling efficiency than helium. Since a fully turbulent boundary layer is established on the model surface, there is no additional heating that is coming from the interaction between the cooling flow and cross flow in the downstream region. In a fully turbulent flow, the cooling efficiency can be remarkably increased by doubled mass flow rate of coolant without inducing any hot spots on the surface. The maximum cooling efficiency of air or argon is less than $20 \%$ even at high mass flow rate, $0.8 \mathrm{~g} / \mathrm{s}$, which corresponds to $2.75 \times 10^{-4}$ blowing ratio. This cooling efficiency is significantly lower compared to a laminar flow, where we can achieve three time higher cooling efficiency with same mass flow rate. However, helium provides a sufficient cooling efficiency in a fully turbulence flow. Using helium as a coolant, we can achieve $60 \%$ cooling efficiency with $0.4 \mathrm{~g} / \mathrm{s}$ mass flow rate, $1.38 \times 10^{-3}$ blowing ratio. The cooling efficiency of helium continuously decreases along the downstream surface, and becomes about $20 \%$ at the end of the PEEK plate. However, we can achieve the $80 \%$ of cooling efficiency by using $0.8 \mathrm{~g} / \mathrm{s}$ mass flow rate which is a factor two higher mass flow rate compared to the laminar flow case.

At both Reynolds numbers, the influence of the cooling with air and argon on the pressure distribution is negligible. The helium cooling film induces slight changes in the pressure distribution.

Acknowledgments This work has been carried out in the frame of DLR research and development activities funded by program directorate Space. The authors would like to thank to the H2 K facility engineer Michael Kosbow and technician Marco Schmors for their support in preparation of the test model and operation of the facility.

Open Access This article is distributed under the terms of the Creative Commons Attribution Noncommercial License which permits any noncommercial use, distribution, and reproduction in any medium, provided the original author(s) and source are credited.
Esser B, Gülhan A (2008) Qualification of active cooling concepts in ground facilities. In: Gülhan A (ed) RESPACE-key technologies for re-usable space systems, NNFM 998. Springer, Berlin, pp 104-131

Esser B, Gülhan A, Kuhn M (2007) Thermal qualification of transpiration cooling for atmospheric entry. In: Proceedings of the 1st CEAS European air and space conference, Berlin, Germany, 10.9. bis 13.9.2007, pp S3205-S3210

Fluent Inc. (2003) Fluent 6.1 user's guide

Ghadiani SR (2005) A multiphasic continuum mechanical model for design investigations of an effusion cooled rocket thrust chamber. $\mathrm{PhD}$ thesis, Universität Stuttgart, Germany

Gülhan A, Schütte G, Stahl B (2008) Experimental study on aerothermal heating caused by jet-hypersonic crossflow interaction. AIAA J Spacecr Rockets 45(5):891-899

Häberle J, Gülhan A (2007) Internal flowfield investigation of a hypersonic inlet at Mach 6 with bleed. J Propuls Power 23(5)

Henckels A, Gruhn P (2005) Study on aerothermal effects of viscous shock interaction in hypersonic inlets. In: Proceedings of the 5th European symposium on aerothermodynamics for space vehicles, pp 553-558

Heufer KA, Olivier O (2008) Experimental and numerical study of cooling gas injection in laminar supersonic flows. AIAA J 46(11)

Kays WM (1972) Heat transfer to the transpired turbulent boundary layer. Int J Heat Mass Transf 15:1023-1044

Kuhn M, Hald H (2008) Application of transpiration cooling hot structures. In: Gülhan A (ed) RESPACE-key technologies for reusable space systems, NNFM 998. Springer, Berlin, pp 82-103

Lezuo MK (1998) Wärmetransport in $\mathrm{H}_{2}$-transpirativ gekühlten Brennkammerkomponenten. PhD thesis, RWTH Aachen, Germany

Luikov AV (1963) Heat and mass transfer in transpired cooling. Int J Heat Mass Transf 6:559-570

Rubesin MW (1956) The influence of surface injection on heat transfer and skin friction associated with the high speed turbulent boundary layer. NACA RM A55L13

Rubesin MW, Pappas CC (1958) An analysis of the boundary layer characteristics on a flat plate with disturbed light-gas injection. NACA TN 4149

Rubesin MW, Pappas CC (1964) Measurement of heat transfer and recovery factor of a compressible turbulent boundary layer on a sharp cone with foreign gas injection. NASA TN D-2230

Serbest E (2002) Untersuchungen zur Anwendung der Effusionskühlung bei Raketenbrennkammern. PhD thesis, RWTH Aachen, Germany

Van Foreest A, Gülhan A, Esser B, Sippel M, Ambrosius B, Sudmeijer K (2007) Transpiration cooling using liquid water. In: 39th AIAA thermophysics conference, paper AIAA 2007-4034, Miami, FL, USA

\section{References}

Delery J (1988) Shock/shock and shock-wave/boundary-layer interactions in hypersonic flows. AGARD-FDP-VKI, 30 